This item was submitted to Loughborough's Research Repository by the author.

Items in Figshare are protected by copyright, with all rights reserved, unless otherwise indicated.

\title{
Optimised configuration of sensors for fault tolerant control of an electro- magnetic suspension system
}

\section{PLEASE CITE THE PUBLISHED VERSION}

http://dx.doi.org/10.1080/00207721.2011.598959

\section{PUBLISHER}

(C) Taylor \& Francis

\section{VERSION}

AM (Accepted Manuscript)

\section{LICENCE}

CC BY-NC-ND 4.0

\section{REPOSITORY RECORD}

Michail, Konstantinos, Argyrios C. Zolotas, Roger M. Goodall, and J.F. Whidborne. 2019. "Optimised Configuration of Sensors for Fault Tolerant Control of an Electro-magnetic Suspension System". figshare. https://hdl.handle.net/2134/9252. 
This item was submitted to Loughborough's Institutional Repository (https://dspace.lboro.ac.uk/) by the author and is made available under the following Creative Commons Licence conditions.

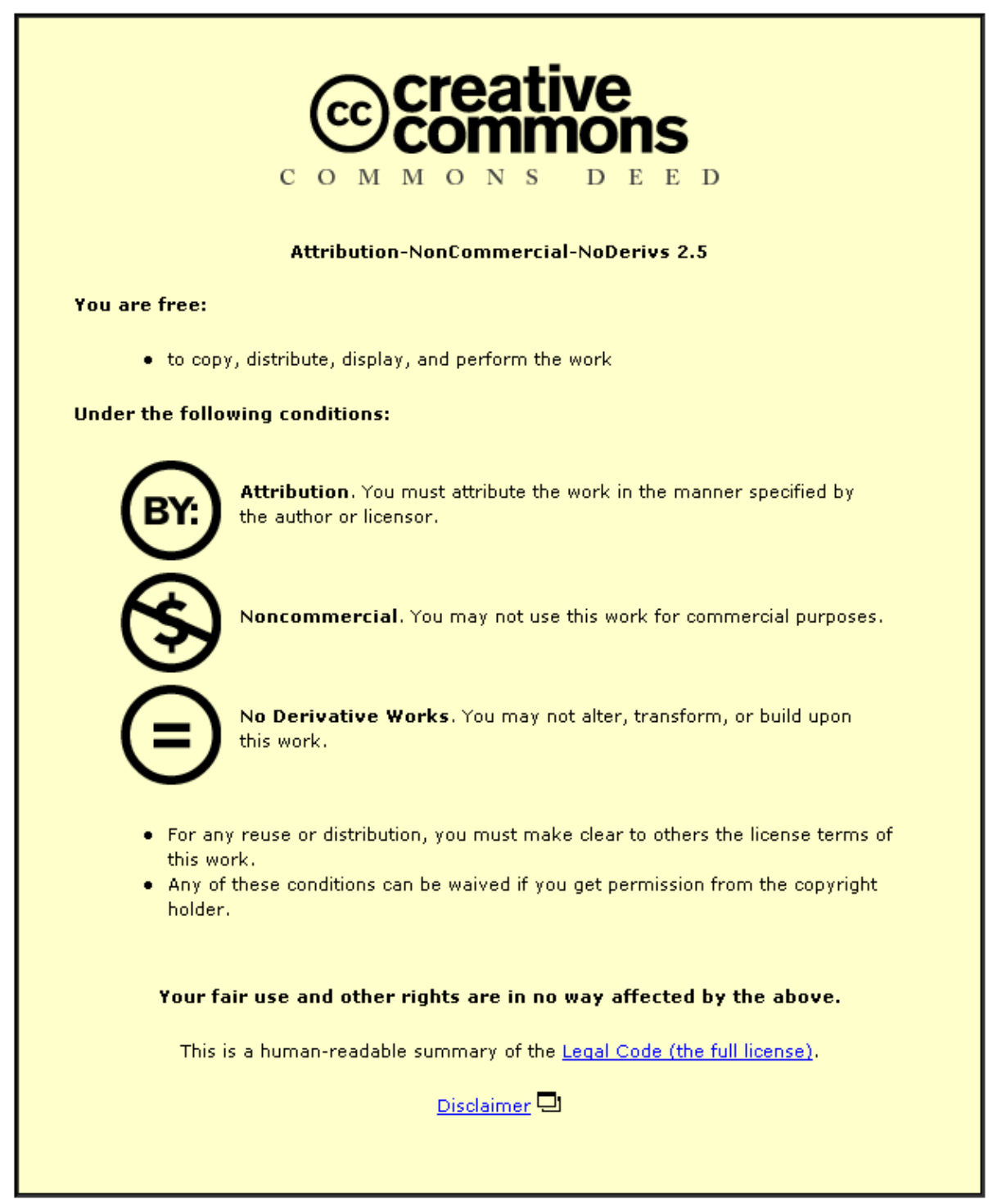

For the full text of this licence, please go to: http://creativecommons.org/licenses/by-nc-nd/2.5/ 


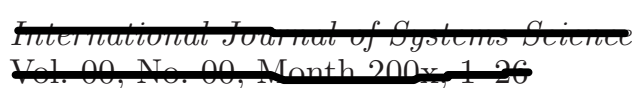

Author's version:

To cite this article: K. Michail, A.C. Zolotas, R.M. Goodall \& J.F. Whidborne (2011): Optimised configuration of sensors for fault tolerant control of an electro-magnetic suspension system, International Journal of Systems Science, DOI:10.1080/00207721.2011.598959

\title{
RESEARCH ARTICLE
}

\section{Optimised Configuration of Sensors for Fault Tolerant Control of an Electro-Magnetic Suspension System}

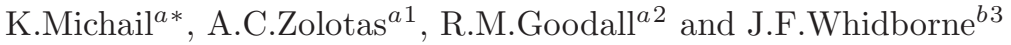 \\ ${ }^{a}$ Control Systems Group, Department of Electrical and Electronic Engineering, Loughborough \\ University, Loughborough, United Kingdom; ${ }^{b}$ Dynamics, Simulation and Control Group, Department of \\ Aerospace Sciences, Cranfield University, Cranfield, United kingdom
}

(Review Version)

\begin{abstract}
For any given system the number and location of sensors can affect the closed-loop performance as well as the reliability of the system. Hence one problem in control system design is the selection of the sensors in some optimum sense that considers both the system performance and reliability. Although some methods have been proposed that deal with some of the aforementioned aspects, in this work, a design framework dealing with both control and reliability aspects is presented. The proposed framework is able to identify the best sensor set for which optimum performance is achieved even under single or multiple sensor failures with minimum sensor redundancy. The proposed systematic framework combines linear quadratic gaussian control, fault tolerant control and multiobjective optimisation. The efficacy of the proposed framework is shown via appropriate simulations on an electro-magnetic suspension system.
\end{abstract}

Keywords: Optimised sensor Configurations; Sensor fault tolerance; Electromagnetic suspension;Genetic Algorithms;LQG control

\section{Introduction}

In recent years, there has been a high demand for technological advances within the automotive, aerospace and other transport sectors. Consequently, it is increasingly important to reduce cost and complexity and to improve the reliability and safety of such technologies. In this context, control systems can play a very important role. Part of the control system design is the selection of the input/outputs (actuators/sensors) to be used for controlling a plant (Wal and Jager 2001) and (for safety-critical) systems offer fault tolerance (Blanke et al. 1997, 2003).

Optimum selection of the inputs/outputs subject to control properties (e.g. optimum performance, minimum cost and complexity, fault tolerant control and robustness) is a non-trivial task especially if the system is characterised by complicated dynamics. To solve this problem, the authors suggest a novel systematic framework which appears to be a simple solution to a rather complicated and until today unresolved problem. In order to present this framework, concepts from control and metaheuristics are merged into the proposed systematic framework. Modern control methods, Fault tolerant control and Genetic Algorithms are combined to develop the proposed framework. The efficacy of the proposed framework is tested on a maglev vehicle suspension.

The suspension of a MAGLEV vehicle is designed to support the mass of the vehicle and the passengers under any circumstances e.g. disturbances and/or faults. Typically, there are broadly

\footnotetext{
*Corresponding author email: kon_michael@ieee.org

1.Email: a.c.zolotas@lboro.ac.uk 2.Email: r.m.goodall@lboro.ac.uk 3.Email: j.f.whidborne@cranfield.ac.uk

ISSN: 0020-7721 print/ISSN 1464-5319 online

(C) 200x Taylor \& Francis

DOI: $10.1080 / 0020772 \mathrm{YY} \times x x x x x x x$

http://www.informaworld.com
} 
three types of such suspensions (Lee et al. 2006): (i) The Electro-Magnetic Suspension (EMS), (ii) Electro-Dynamic Suspension (EDS) and (iii) Hybrid electromagnetic suspension. For the first two types of suspensions, electromagnets are used while in the latter case combination with permanent magnets and electromagnets achieve the desired suspension characteristics. The EMS system consists of an electromagnet where the mass of the vehicle and the passengers are supported. The levitation is achieved by producing an attractive force onto the rail and, by controlling the circulated flux, the airgap (distance between the electromagnet and the rail) can be controlled. The EMS system is a critical part of the MAGLEV vehicle and in fact, is nonlinear, inherently unstable and safety-critical system with non-trivial set of control requirements as described by Goodall (1994, 2004).

Even though the EMS system has only one control input (electromagnet), selecting the outputs (sensors) in an optimum manner is a complex and time consuming process to do manually. Therefore, the proposed systematic framework can be used to simplify the sensor selection process for the suspension. Optimum sensor selection for single stage EMS systems has not been considered before and this can serve as a baseline for larger scale complex plants.

Linear Quadratic Gaussian (LQG) control is a well known modern control method that has been implemented in many control applications where the state estimation and noise filtering are essential requirements. The LQG controller design combines a Linear Quadratic Regulator (LQR) feedback controller and Kalman-Bucy Estimator (KBE). The LQR controller is based on state feedback control (see Anderson and Moore (1990), Friedland (1986), Maciejowski (1990)) while the KBE introduced by Kalman and Bucy (1961) is used for the state estimation and noise filtering. Skogestad and Postlethwaite (2005) shows that the LQG controller is designed according to the separation principle. Firstly, tuning of the LQR controller is done followed by the KBE tuning. The second part involves the selection of the appropriate measurements which which a good state estimation and noise filtering are achieved. Under this scope the the proposed framework can be used to select the best measurements for the LQG controller performance. Safety-critical systems require mechanism to accommodate faults. This is because faults can lead to instability which can thread the integrity of such system (e.g. the MAGLEV vehicle, airplane etc). Faults, are unavoidable therefore the Fault Tolerant Control (FTC) concept is used in many safety-critical systems. The FTC design aims to maintain the performance of the system after a fault occurs (Blanke et al. 1997). Typically two approaches exist, the Passive FTC (PFTC) and the Active FTC (AFTC). The second approach which is used in the framework requires a priori knowledge about the possible faults that may occur or a mechanism to detect and isolate any unanticipated faults. In any case, the controller structure has to change in order to accommodate the faults. For the PFTC approach the design of the controller ensures robustness against the faults i.e. when faults happen the performance is maintained without any change of the controller structure because of the insensitivity to those faults (Patton 1997).

An optimisation tool has to be used in order to achieve multiple system control properties. The metaheuristic optimisation algorithms are very strong optimisation tools and they been used in many disciplines (Dreo et al. 2006). Various types of such algorithms exist among them the Evolutionary Algorithms (EAs) that are successfully merged in the proposed framework. EAs are well-known and efficient optimisation tools extensively used in control engineering design because they are able to handle multiple conflicting objectives and many variables in a very efficient manner (Fleming and Purshouse 2002). However they haven't been tested under the scope of sensor optimisation as in this paper. Various versions of EAs have been developed since the first introduction by Goldberg (1989). These are well summarised by Konak et al. (2006) with the most recent version published by Zou et al. (2008). In this paper the recently developed Non-dominated Sorting Genetic Algorithm-II (NSGA-II) is used. NSGA-II has been developed by Deb et al. (2002) and it has been shown to be a powerful optimisation tool in multiobjective optimisation problems. Details of the particular Genetic Algorithm (GA) can be found in Michail (2009) where rigorous explanation along with examples using appropriate test functions are presented. 
The novelty in this paper relies on the fact that LQG control, fault tolerant control and tuning via genetic algorithms concepts are combined to form a systematic framework in an attempt to simplify the "best" selection of sensor sets for the EMS system subject to optimum closed-loop performance and ensuring integrity of the system under possible sensor faults. In this context, the algorithm explores and separately optimise the performance of the EMS using all feasible sensor sets in order to identify fallback options under single or multiple sensor faults, i.e. instants of one or multiple sensors failing stability constraints but with optimum performance maintained by controller reconfiguration using the remaining healthy sensors.

The rest of the paper is organised as follows: Section 2 presents the need to have a systematic framework for optimum sensor/actuator selection and the description of the proposed framework to do that. Section 3 gives a rigorous description of a typical dynamically complex application like the EMS system. The unstable dynamic non-linear model is given along with the linearized model, control requirements and all possible sensor sets. Section 4 describes the multiobjective constrained optimisation that is vital for the implementation of the framework. A short description of the NSGA-II and constrained handling techniques is given. In Section 5 the classical control strategies are described which are creating a baseline for the development of the systematic framework. An insight is given and the NSGA-II efficacy is tested under multiple non-trivial requirements. The usefulness of the constraint handling techniques is also highlighted in the same section. Section 6 describes the LQG control formulation for the EMS system as is used within the systematic framework and in Section 7 the efficacy of the proposed framework is assessed via appropriate simulations on the EMS. Finally, in Section 8 the conclusion from the simulation and data analysis is extracted.

\section{Which Sensor Set?}

Any given plant in nature has a number of control inputs $\left\{u_{i}: i=1, \ldots n_{u}\right\}$, input disturbances $\left\{d_{i}: i=1, \ldots n_{d}\right\}$ and a set of possible outputs (sensors), $\mathcal{Y}=\left\{y_{i}: i=1, \ldots n_{s}\right\}$. The problem is to determine the set of sensors, $\mathcal{Y}_{\text {opt }} \subset \mathcal{Y}$, for which the system

(1) satisfies a set of closed-loop performance criteria,

(2) satisfies a set of fault tolerance criteria,

(3) the sensor set has minimum redundancy i.e. the number of elements in $\mathcal{Y}_{\text {opt }}$ is minimal,

(4) has sufficient robustness against uncertainties and

(5) has low cost

The selection of $\mathcal{Y}_{\text {opt }}$ subject to the aforementioned properties is a very important and complex process especially if the plant has a lot of actuator/sensor configuration possibilities. The problem becomes more complicated if the plant has real, non-trivial set of requirements with inherently unstable and non-linear characteristics. The work in this paper is focused upon the optimum sensor selection of the EMS subject to the first three properties. Nevertheless, the proposed frameworks is very flexible and can be extended to include optimum selection of both actuators and sensors subject to all of the aforementioned properties.

Figure 1 shows a typical control system diagram which illustrates the sensor selection problem. The sensor set $\left(\mathcal{Y}_{\text {opt }}\right)$ is a subset of the full sensor set $(\mathcal{Y})$. This is selected among other candidates in order to control the plant with optimum performance. Obviously, there are many subsets of the full sensor set and the number of them can be calculated from

$$
N_{s}=2^{n_{s}}-1
$$

where, $N_{s}$ is the total number of all sensor sets (having from 1 to $n_{s}$ sensors) and $n_{s}$ the total number of sensors.

Moreover, safety-critical plants require fault tolerance for sensor failures therefore the selection of the "best" sensor set has to consider sensor fault tolerance as well with the minimum number 
of sensors. This means that when one or more sensors fail the performance of the plant should be maintained by using the remaining healthy sensors, $\mathcal{Y}_{h}$ i.e. $\mathcal{Y}_{h} \subset \mathcal{Y}_{\text {opt }}$. In this context a bank

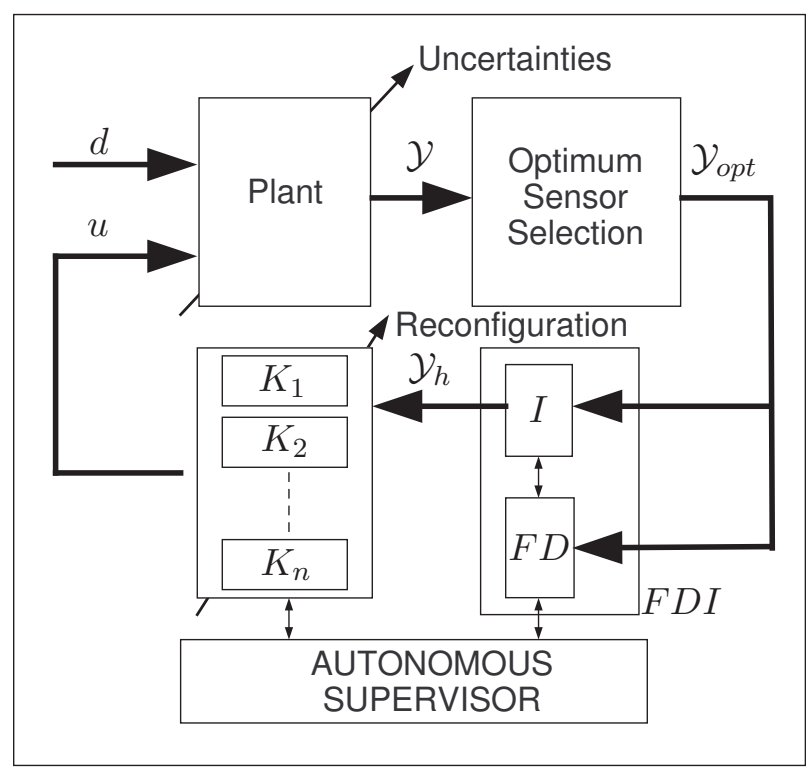

Figure 1. Block diagram for optimum sensor selection for control and fault tolerance.

of controllers is saved and when sensor faults happen the performance of the suspension is maintained via controller reconfiguration. This is where the AFTC concept comes in to assist. When one or more sensors fail the faulty sensors are removed from the loop and the performance is maintained by switching to the appropriate controller (i.e. controller reconfiguration). The controller is optimally tuned for the remaining healthy sensor set $\mathcal{Y}_{h}$ ensuring optimum closed-loop performance even under sensor faults (note that the performance of the system is optimised for all candidate sensor sets). In order to achieve controller reconfiguration a Fault Detection and Isolation (FDI) mechanism is required. The aim of the FDI is: (i) To detect multiple sensor faults (ii) isolate the faulty sensors.

Finally, the question in hand is posed as "Which is the optimum sensor set, $\mathcal{Y}_{\text {opt }}$ that is able to control a complex dynamical system in a desired manner while offering sensor fault tolerance with minimum redundancy?" This question is answered using the proposed framework. The framework is able to identify the minimum number of sensors that can be used for control and fault tolerance and thus provide a cheaper and safer control system. In order to do that, it combines the LQG control and the NSGA-II in order to optimally tune the closed-loop performance for each feasible sensor set of the EMS system. Additionally, the Fault Tolerant Control concept is introduced in the framework for sensor fault tolerance with minimum sensor redundancy.

The overall sensor optimisation process is done in two steps: (i) the LQR controller is tuned and the Pareto-optimality between the multiple objectives is found. In this step, the controller (i.e. state feedback gains) which results to the desired closed-loop response is selected as the 'ideal' or reference response for the tuning of the KBE that is done next. (ii) The KBE is optimally tuned for every feasible sensor set in order to achieve the 'ideal' closed-loop response. At the end of the second part a table with optimised sensor configurations is given where the 'best' sensor set is selected. The flowchart of the proposed framework is depicted in Fig. 2. The execution of the algorithm is done in the following way:

Algorithm initialisation: The GA parameter assignment (for the NSGA-II these parameters are listed on Table 3), the objective functions to be minimized in (9), the control constraints listed on Table 2 and the user's controller selection criteria $\left(f_{c_{i}}, f_{u}\right)$ are the inputs to the algorithm. 


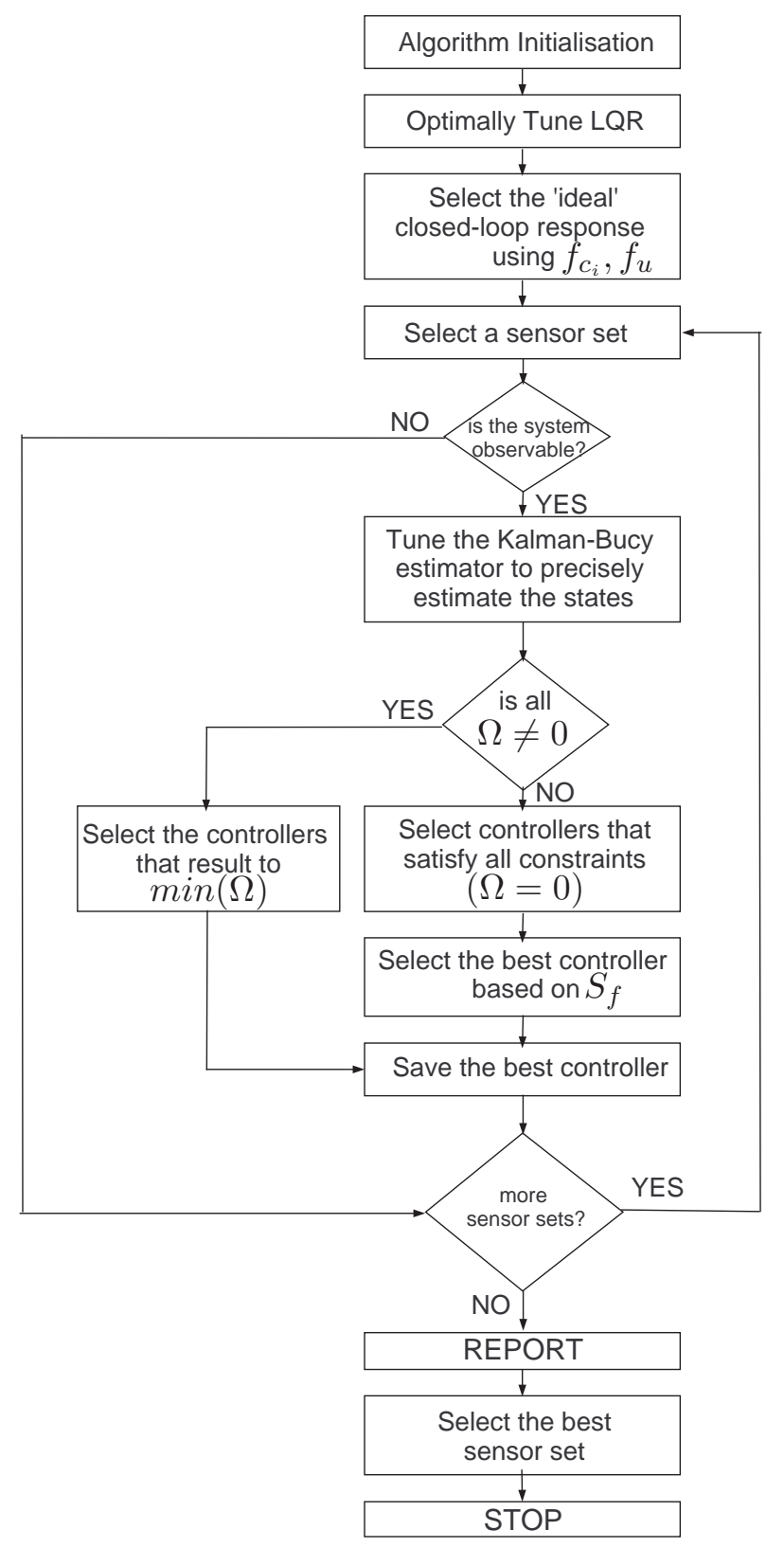

Figure 2. Flowchart of the proposed sensor optimisation systematic framework.

Optimum LQR tuning: Provided that controllability is achieved the LQR controller is optimally tuned in order to find the Pareto-optimality between the objective functions using NSGA-II. At this stage the 'ideal' closed-loop response is selected based on $f_{c_{i}}$ and $f_{u} \cdot f_{c_{i}}$ criteria ensure that the selected controllers result to the desired closed-loop characteristics and $f_{u}$ criterion makes sure that the selected controller results to the 'ideal' closed-loop response.

Sensor set feasibility:At this stage the sensor feasibility is considered. The sensor set is selected and the state observability is tested. If the state observability is not possible using the current sensor set then the algorithm moves to the next. When the state observability is achieved optimum tuning of the KBE is considered next.

Optimum Kalman-Bucy tuning: At this stage the KBE is optimally tuned to achieve the 'ideal' response for the current sensor set. After this step a number of controllers (equal to the number of NSGA-II population) are available from where the best one will be selected. 
Best controller selection: During this step the best controller which results to a closed-loop response as close as possible to the 'ideal' one is selected. For this purpose two metrics are used: (i) the overall constraint violation function $(\Omega)$ and (ii) the state estimation error function $\left(S_{f}\right)$. The first, is used to select those controllers that satisfy the control constraints as explained in Section 5.1. However, if there is no controller exist to satisfy the control constraints (i.e. all resulting $\Omega \neq 0$ ) then the one with the smallest $\Omega$ is selected. In the opposite case (i.e. those controllers that result to $\Omega=0$ ) where the group of controllers that satisfy the control constraints are selected and the one with the smallest state estimation error $S_{f}$ is selected as the best controller. The state estimation error function is the error between the estimated and the 'ideal' states (for details see Section 6.2). At the end the rationale is that the controller which results to the closed-loop response closest to the 'ideal' is selected.

Optimum sensor set selection: The next step is to optimally tune the KBE for every feasible sensor set. At this stage the selection of the best sensor set $\left(\mathcal{Y}_{\text {opt }}\right)$ for control and sensor fault tolerance is done.

\section{The EMS System application}

\subsection{The EMS System model}

In this section the EMS system modelling is described. The non-linear model, the linearized state space, the disturbance inputs and the closed-loop control requirements are analysed.

The 1DOF (degree-of-freedom) model represents the quarter of a typical MAGLEV vehicle. The basic quarter car model of the MAGLEV vehicle is shown in Fig. 3. This is a single-stage EMS that has been shown to be suitable for low speed vehicle (Goodall 2004), where other MAGLEV systems, particularly those for high speed, have a separate conventional secondary suspension for proper ride quality. The suspension consists of an electromagnet with a ferromagnetic core that is attracted to the rail which is also made out of ferromagnetic material. The electromagnet has a pole face area $A_{p}$, and a coil of $N_{c}$ turns. The carriage mass is attached to the electromagnet giving a total suspended mass of $M_{s}$, with $z_{t}$ the rail's position and $z$ the electromagnet's position. The airgap $\left(z_{t}-z\right)$ is to be maintained close to the operating condition required (15mm in this case). As described by Goodall $(2008,1985)$ there are four important variables in

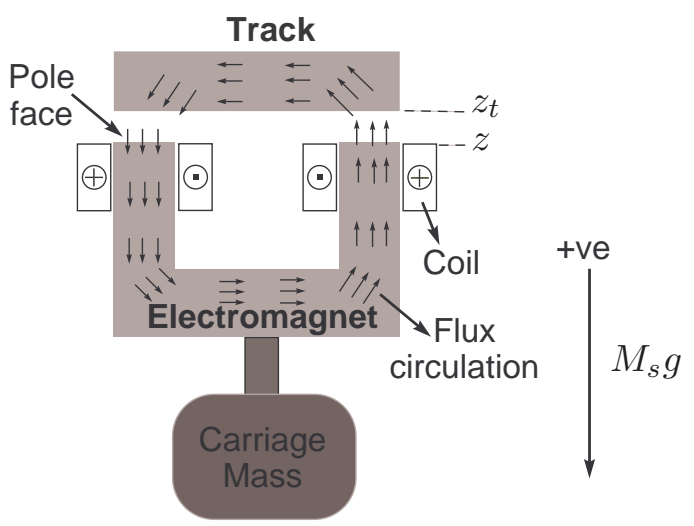

Figure 3. Typical diagram of a single stage EMS System.

an electromagnet named as force $F$, flux density $B$, airgap $G$ and the coil's current $I$ that give the non-linear characteristics to the suspension.

Assuming that the motion vertically downwards is taken as positive the non-linear model of the EMS is described by Newton's equation of motion (2) and the electrical circuit involved from the electromagnet's coil (3). Equations in (4) describe the flux density and the force. The airgap 
velocity is given in (5) (Michail 2009).

$$
\begin{aligned}
& M_{s} \frac{d^{2} Z}{d t^{2}}=M_{s} g-F \\
& V_{c}=I R_{c}+L_{c} \frac{d I}{d t}+N_{c} A_{p} \frac{d B}{d t} \\
& B=K_{b} \frac{I}{G}, F=K_{f} B^{2} \\
& \frac{d G}{d t}=\frac{d z_{t}}{d t}-\frac{d Z}{d t}
\end{aligned}
$$

where, $Z$ is the electromagnet's position, $g$ the gravity acceleration taken as $9.81 \mathrm{~m} / \mathrm{s}^{2}, V_{c}$ the coil input voltage, $R_{c}$ coil's resistance, $L_{c}$ coil's inductance, $K_{b}$ the flux constant and $K_{f}$ force constant.

The linearisation of the EMS system is based on small perturbations around the operating point. The lower case letter denotes the small variation around the operating point and subscript ' $\mathrm{O}$ ' represents the operating point, e.g. $I=I_{o}+i$. Also note that the airgap is taken as $G=$ $G_{o}+\left(z_{t}-z\right)$. As described by Michail (2009) the linearized model of the EMS can be expressed in state space form (6). The states are $x=\left[\begin{array}{lll}i & \dot{z} & \left(z_{t}-z\right)\end{array}\right]^{T}$ and the output equation corresponds to the following five measurements: the coil's current $i$, the flux density $b$, the airgap $\left(z_{t}-z\right)$, the vertical velocity $\dot{z}$ and the vertical acceleration $\ddot{z}$. The matrices $A, B_{u}, B_{\dot{z}_{t}}$ and $C$ are given in (7) and (8).

$$
\begin{aligned}
& \dot{x}=A x+B_{u} u_{c}+B_{\dot{z}_{t}} \dot{z}_{t} \\
& y=C x \\
& A=\left[\begin{array}{ccc}
-\frac{R_{c}}{L_{c}+\frac{K_{b} N_{c} A_{p}}{G_{o}}}-\frac{K_{b} N_{c} A_{p} I_{o}}{G_{o}^{2}\left(L_{c}+\frac{K_{b} N_{c} A_{p}}{G_{o}}\right)} & 0 \\
-2 K_{f} \frac{I_{o}}{M_{s} G_{o}^{2}} & 0 & 2 K_{f} \frac{I_{o}^{2}}{M_{s} G_{o}^{3}} \\
0 & -1 & 0
\end{array}\right] \\
& B_{u}=\left[\begin{array}{c}
\frac{1}{L_{c}+\frac{K_{b} N_{c} A_{p}}{G_{o}}} \\
0 \\
0
\end{array}\right], B_{\dot{z}_{t}}=\left[\begin{array}{c}
\frac{K_{b} N_{c} A_{p} I_{o}}{G_{o}^{2}\left(L_{c}+\frac{K_{b} N_{c} A_{p}}{G_{o}}\right)} \\
0 \\
1
\end{array}\right], C=\left[\begin{array}{ccc}
1 & 0 & 0 \\
\frac{K_{b}}{G_{o}} & 0 & -\frac{K_{b} I_{o}}{G_{o}^{2}} \\
0 & 0 & 1 \\
0 & 1 & 0 \\
-2 K_{f} \frac{I_{o}}{M_{s} G_{o}^{2}} & 0 & 2 K_{f} \frac{I_{o}^{2}}{M_{s} G_{o}^{3}}
\end{array}\right]
\end{aligned}
$$

The sensor combinations (or sets) can be easily obtained by using the output matrix $C$ in (8). The total number of sensor sets can be calculated from (1). Given that the EMS system has 5 outputs there are 31 candidate sensor sets from where the $\mathcal{Y}_{\text {opt }}$ should be identified using the proposed framework.

A typical quarter car vehicle of $1000 \mathrm{~kg}$ requires a nominal force of $F_{o}=M_{s} \times g$ and the nominal airgap $\left(G_{o}\right)$ is set at $15 \mathrm{~mm}$ to accommodate the track roughness. According to these requirements the rest of the suspension parameters listed on Table 1 can be calculated. Details on the design of electromagnets for MAGLEV vehicles are given by Goodall (1985). 


\begin{tabular}{llll} 
Table 1. Parameters of the Electro-magnetic suspension. & \\
\hline \hline Parameter & Value & Parameter & Value \\
\hline Operating airgap, $G_{o}$ & $0.015 \mathrm{~m}$ & Carriage Mass, $M_{s}$ & $1000 \mathrm{~kg}$ \\
Operating flux density, $B_{o}$ & $1 \mathrm{~T}$ & Coil's Resistance, $R_{s}$ & $10 \Omega$ \\
Operating current, $I_{o}$ & $10 \mathrm{~A}$ & Coil's Inductance, $L_{c}$ & $0.1 \mathrm{H}$ \\
Operating voltage, $V_{o}$ & $100 \mathrm{~V}$ & Number of turns, $N_{c}$ & 2000 \\
Operating force, $F_{o}$ & $9810 \mathrm{~N}$ & Pole face area, $A_{p}$ & $0.01 \mathrm{~m}^{2}$ \\
\hline \hline
\end{tabular}

\subsection{Input disturbances to the suspension}

\subsubsection{Deterministic disturbances}

The main deterministic inputs to the suspension in the vertical direction are the transitions onto track gradients. In this paper, the deterministic input is a track gradient of $5 \%$ at a vehicle speed of $15 \mathrm{~m} / \mathrm{s}$ and a vertical acceleration of $0.5 \mathrm{~m} / \mathrm{s}^{2}$ with jerk level of $1 \mathrm{~m} / \mathrm{s}^{3}$.

\subsubsection{Stochastic disturbances}

Stochastic disturbance is the random inputs to the EMS system in the vertical direction. Random inputs represent the inaccuracies of laying the rail, the lack of straightness of the rail and the effects of fixtures. Two models of the stochastic track behaviour are presented by Paddison (1995). The velocity variations in the vertical direction are quantified by a doublesided power spectrum density which in the frequency domain is expressed by $S_{\dot{z}_{t}}=\pi A_{t} V_{V}$. $V_{V}$ is the vehicle's speed and $A_{t}$ is the track roughness taken as $15 \mathrm{~m} / \mathrm{s}$ and $1 \times 10^{-7} \mathrm{~m}$ (for high quality rail) respectively. The corresponding (one-sided) autocorrelation function is given by $R(\tau)=2 \pi^{2} A_{t} V_{V} \delta(\tau)$. Regardless a linear controller is used for the control, the simulations are actually based on the implementation of the controller to the nonlinear EMS system (see Friedland (1996)). Hence, the RMS values of the required quantities (acceleration, current, input voltage etc) are quantified using time history data.

\subsection{Design requirements}

The control requirements of an EMS system depend on the type and speed of the MAGLEV vehicle. The dynamic characteristics of an EMS are described by Goodall (1994, 2004). The description is focused upon the EMS of the low speed Birmingham Airport Maglev vehicle requirements which operated successfully in the UK for more than 10 years. Table 2 tabulates the closed-loop control limitations for the deterministic and stochastic features. Fundamentally, there is a trade off between the deterministic and the stochastic (ride quality) responses of an EMS system. In this case, the deterministic response is limited to the maximum standard values while the stochastic features have been set as objective functions to be minimized, i.e minimize the vertical acceleration (improve ride quality) and the RMS current variations (input current). These objectives can be formally given as

$$
\phi_{s_{1}}=i_{r m s}, \quad \phi_{s_{2}}=\ddot{z}_{r m s}
$$

Additionally, in any real application sensors add noise to the measured quantities. Although the dynamical behaviour of the EMS system is similar to a low pass filter, the noise coming from sensors could be amplified by the controllers if not taken into account in the design (Michail 2009). Thus an upper limit on the RMS of the noise in the actuator signal is introduced and is set at $50 V_{r m s}$. The noise covariance for each sensor is taken as $1 \%$ of the maximum value of the deterministic response for the corresponding measured quantity. 
Table 2. Control constraints for the Electro-Magnetic Suspension closed-loop performance.

\begin{tabular}{l|l}
\hline \hline Measurement & Value \\
\hline Response with stochastic track profile & \\
RMS acceleration, $\ddot{z}_{r m s}$ & $\leq 0.5 \mathrm{~ms}^{-2}$ \\
RMS airgap variation, $\left(z_{t}-z\right)_{r m s}$ & $\leq 5 \mathrm{~mm}$ \\
RMS control effort, $u_{c_{r m s}}$ & $\leq 300 \mathrm{~V}$ \\
\hline Response with deterministic track profile & \\
Maximum airgap deviation, $\left(z_{t}-z\right)_{p}$ & $\leq 7.5 \mathrm{~mm}$ \\
Maximum control effort, $u_{c_{p}}$ & $\leq 300 \mathrm{~V}$ \\
Settling time, $t_{s}$ & $\leq 3 \mathrm{~s}$ \\
Airgap steady state error, $e_{\left(z_{t}-z\right)_{s s}}$ & $=0$ \\
\hline Response with idle track profile & \\
RMS of the noise on control effort, $u_{n_{r m s}}$ & $\leq 50 \mathrm{~V}_{r m s}$ \\
\hline \hline
\end{tabular}

\section{Multi-objective constrained optimisation}

The problem described in Section 2 requires a powerful optimisation tool especially if the performance is to be optimised using all sensor sets in an iterative manner. In this Section a brief description of the NSGA-II (Deb et al. 2002) is done along with the constraint handing techniques. Constraint handling techniques are used not only to ensure that the closed-loop performance of the EMS is within limits but they also serve as a controller selection criterion as described in Section 5.1. In this paper, the NSGA-II is used in three cases where different parameter assignment is required in each case. Proper assignment of those values is vital because fast convergence and good spread of the solutions onto the optimum Pareto front depends on them. There is no generalised way for deciding about the values of the NSGA-II parameters but they can be selected from experience as it is done in this work. The assigned parameters for the classical control strategies and the proposed systematic framework are tabulated on Table 3. The generation number is defined as the stopping criterion and population size is the number of chromosomes that the population, $n_{c}$, consists of. The crossover probability is generally selected to be large for good mixing of genetic material and the mutation probability is a function of the number of variables $n_{V}$.

Because this problem requires a lot of control constraints to be simultaneously achieved a

Table 3. NSGA-II parameters for the optimisation of Classical
control and the proposed systematic framework.
\begin{tabular}{lccc}
\hline \hline Parameter & CC & LQR & Kalman-Bucy \\
\hline Maximum generation & 500 & 500 & 50 \\
Population size, $n_{c}$ & 70 & 50 & 20 \\
Crossover probability & 0.9 & 0.9 & 0.9 \\
Mutation probability & $1 / n_{V}$ & $1 / n_{V}$ & $1 / n_{V}$ \\
\hline \hline
\end{tabular}

constraint handling technique is necessary. Various constraint handling techniques exist in the literature among them the penalty functions that are used here. There are many versions of penalty functions but only two approaches are useful in this paper. One is the death penalty approach and the other the static penalty approach (Coello 2002). The purpose of using penalty functions is twofold: (i) To ensure that the closed-loop responses of the stochastic and deterministic inputs are within the limitations as described in Section 3.3 and (ii) To use the overall constraint violation function in (12) for selecting appropriate controllers as explained latter in Section 5.1.

The static penalty method is rigorously described by Deb (2001) and summarised as follows The $i^{\text {th }}$ soft constraint violation $\omega_{i}$, is given as

$$
\omega_{i}\left(k^{i}\right)= \begin{cases}\left|g_{l_{i}}\left(k^{i}\right)\right|, & \text { if } g_{i}\left(k^{i}\right) \geq 0 \\ 0 & \text { otherwise }\end{cases}
$$

where, $k^{i}$ is the $i^{\text {th }}$ measured variable. The constraint violation, $g_{l_{i}}\left(k^{i}\right)$, is given as $g_{l_{i}}\left(k^{i}\right)=$ $-\left(k^{i} / k_{d}^{i}\right)+1$ where $k_{d}^{i}$ is the maximum allowed desired value of $k^{i}$. For the $i^{t h}$ values constrained 
to be higher than the minimum allowed, the constraint violation is given as $g_{h_{i}}\left(k^{i}\right)=\left(k^{i} / k_{d}^{i}\right)-1$. The hard constraints are those whose values are not allowed to deviate from the predefined ones i.e. the steady state error of the airgap must be equal to zero. The $j^{\text {th }}$ hard constraint violation, $\psi_{j}$, for each measured variable $f^{j}$, is given as

$$
\psi_{j}\left(f^{j}\right)= \begin{cases}\left|h_{j}\left(f^{j}\right)\right| & \text { if } h_{j}\left(f^{j}\right) \neq 0 \\ 0, & \text { otherwise }\end{cases}
$$

However, this can transformed into a soft constraint, allowing a small tolerance value $\xi$ (Coello 2002). The hard constraint violation $h_{j}\left(f^{j}\right)$, function is then calculated as $h_{j}\left(f^{j}\right)=\left|f^{j}\right|-\xi<0$. The overall constraint violation function $\Omega\left(k^{(i)}, f^{(j)}\right)$, is then derived as

$$
\Omega\left(k^{(i)}, f^{(j)}\right)=\sum_{i=1}^{i} \omega_{i}\left(k^{(i)}\right)+\sum_{j=1}^{j} \psi_{j}\left(f^{(j)}\right)
$$

$\Omega$ is then added to each one of the objective functions $\omega$ forming the following penalty equation

$$
\Phi_{t}=\phi_{t}+R_{t} \Omega\left(k^{(i)}, f^{(j)}\right)
$$

where $\phi_{t}$ is the $t^{t h}$ objective function, $\Phi_{t}$ is the $t^{t h}$ penalised objective function and $R_{t}$ is the $t^{t h}$ penalty parameter from (13). It can be seen that if all constraints are satisfied then $\Omega$ in (12) is zero therefore $\Phi_{t}=\phi_{t}$ while if there is some constraint violation (i.e. $\Omega\left(k^{i}, f^{j}\right) \neq 0$ ) this is added to the objective functions reducing the possibilities for the corresponding solution to survive in the next generations. In this way the objective functions are 'guided' towards the Pareto-optimality with satisfying control constraints. Additionally, $\Omega$ is to be used as a metric for the controllers' performance towards the given constraints in the real control system design problem as described in Section 2.

\section{An Insight to Optimised Configuration of Sensors by using Classical Control}

Although classical control structures for suspensions have been studied previously by Goodall (2000) the same strategies are used here and they are optimised using the Genetic algorithm. The aim of this section is: (i) to illustrate the efficacy of the NSGA-II for optimal tuning of the controllers in a multiobjective framework and (ii) to use the classical control strategies as a baseline for further investigation utilizing modern control and fault tolerant strategies.

The use of inner loops with classical control strategies is advantageous in controlling an EMS system as indicated by Goodall (2000). Figure 4 illustrates the two control configurations compared: (i) with the flux density measurement for the inner loop and the airgap in the outer loop, (ii) current for inner loop and airgap for outer. Control of the non-linear model is achieved by using linear controllers that are designed based on the linear model of the suspension (Friedland 1996). The control problem of the suspension is posed in a multiobjective constrained optimisation framework where the closed-loop performance of the EMS system is optimised subject to a number of control constraints tabulated on Table 2. In order to have sufficient robustness properties, the inner loop bandwidth is constrained between $50 \mathrm{~Hz}-100 \mathrm{~Hz}$ (Goodall 2000). This can be achieved with a Proportional-Integral (PI) controller with a structure as given in (14). The desired outer loop bandwidth of the two control configurations is constrained to be less than $20 \mathrm{~Hz}$ and the phase margin is constrained between the range of $35^{\circ}-45^{\circ}$. Both, control properties can give adequate performance and they are adjusted from direct tuning of the Phase 


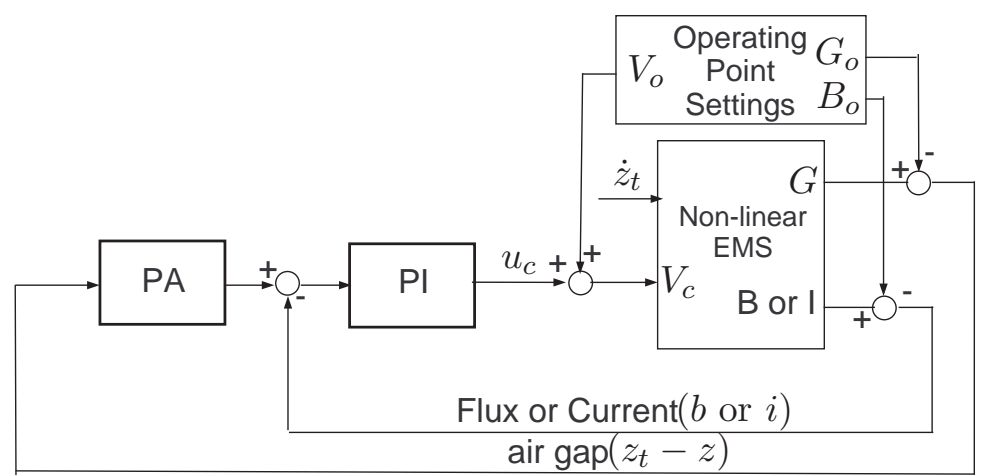

Figure 4. Classical controller implementation with inner loop feedback control using either $\left\{\left(z_{t}-z\right), i\right\}$ or $\left\{\left(z_{t}-z\right), b\right\}$

Advance (PA) controller in (14).

$$
P I=G_{P I} \frac{\tau_{i} s+1}{\tau_{i} s}, \quad P A=G_{P A} \frac{k_{o} \tau_{o} s+1}{\tau_{o} s+1}
$$

The control constraints of the EMS combine both frequency and time responses in order to achieve proper robustness and performance of the suspension. Considering the control performance requirements described in Section 3.3 as well as the robustness properties discussed here, these clearly pose a multiobjective constrained optimisation problem that aims to improve the EMS control by minimizing both objectives in (9) subject to the control constraints listed on Table 2.

NSGA-II is employed to find the Pareto-Optimality between the objectives while ensuring all constraints are satisfied. Undoubtedly designer experience is advantageous in defining initial parameters of the GA that remain constant during the optimisation process. Those parameters are given on Table 3. The mutation probability is defined by the number of the variables to be tuned $\left(n_{V}\right)$, (five according to (14)).

Figure 5 depicts the Pareto-optimality (i.e. the optimum trade-off) between the ride quality $\left(\ddot{z}_{r m s}\right)$ and the RMS coil current $\left(i_{r m s}\right)$ for each sensor configuration, i.e the airgap(outer)/flux(inner) $\left\{\left(z_{t}-z\right), b\right\}$ and the air-gap(outer)/current(inner) $\left\{\left(z_{t}-z\right), i\right\}$ cases. For the former configuration, the vertical acceleration $\ddot{z}_{r m s}$, is limited to the desired value of $0.5 \mathrm{~m} / \mathrm{s}^{2}$ while for the latter configuration is out of the design limitations i.e. $\ddot{z}_{r m s} \leq 5 \mathrm{~m} / \mathrm{s}^{2}$. Therefore, a set of controllers can be selected that satisfy all constraints for the $\left\{\left(z_{t}-z\right), b\right\}$ case but not for the $\left\{\left(z_{t}-z\right), i\right\}$. Furthermore, a detailed comparison between the two sensor configurations is detailed on Table 4 . For the $\left\{\left(z_{t}-z\right), i\right\}$ configuration the vertical acceleration and PM limits are violated in contrast to the $\left\{\left(z_{t}-z\right), b\right\}$ configuration where all control constraints are within the predefined limits. The situation here is simple because there exist only two sensor configurations to select from. In case where many sensor configurations exist with each one having a trade-off similar to the ones in Fig . 5 the optimum sensor selection with the desired performance is difficult thus a criterion is needed to simplify the sensor selection. In the next section, the usefulness of the overall constraint violation function as a controller selection criterion is demonstrated.

\subsection{The overall constraint violation function as a controller selection criterion}

The process of selecting a controller with desired closed-loop characteristics is rather simplified in this case where only two sensor configurations are compared. This can be seen from Table 4 where the $\left\{\left(z_{t}-z\right), i\right\}$ configuration violates two constraints therefore $\left\{\left(z_{t}-z\right), b\right\}$ configuration results to a desired performance. The problem becomes more complex when a large number of sensor configurations (or sensor sets) have to be optimised from where the one that results to the best performance is to be identified. Additionally, for each sensor set, a number of optimally 


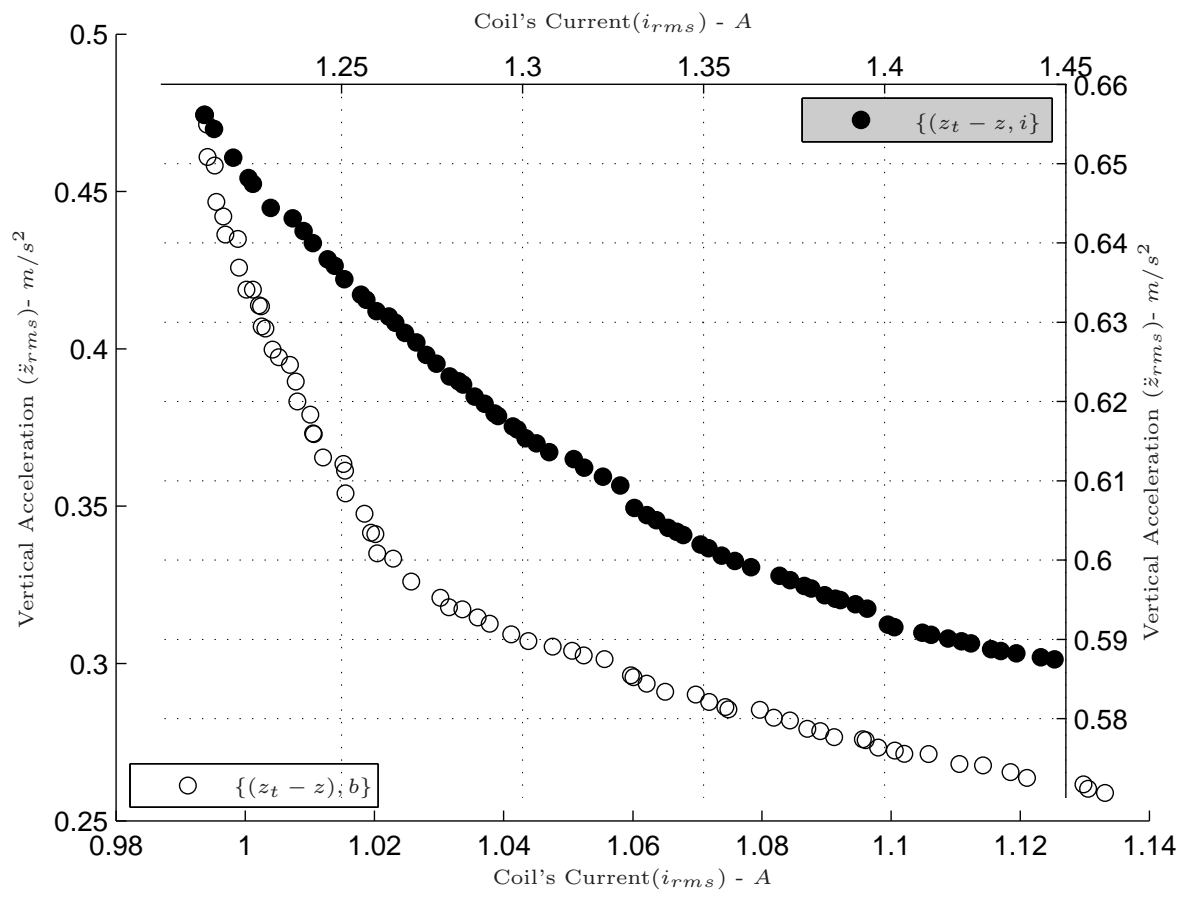

Figure 5. Pareto-optimality of the two classical control strategies (Pareto-Optimality with top-right axes and dark dots corresponds to $\left\{\left(z_{t}-z\right), i\right\}$ and the one with bottom-left axes and white dots to $\left.\left\{\left(z_{t}-z\right), b\right\}\right)$.

\begin{tabular}{lcccc} 
Table 4. Classical control - constraints values for each design. \\
\hline \hline Constraints & $\begin{array}{c}\text { Assigned } \\
\text { values }\end{array}$ & $\left\{\left(z_{t}-z\right), b\right\}$ & $\left\{\left(z_{t}-z\right), i\right\}$ & Units \\
\hline RMS acceleration, $\ddot{z}_{r m s}$ & $\leq 0.5$ & $0.26-0.47$ & $\mathbf{0 . 5 8 - 0 . 6 5}$ & $\mathrm{ms}^{-2}$ \\
RMS airgap, $(z t-z)_{r m s}$ & $\leq 5$ & $1.26-1.53$ & $1.4-1.7$ & $m m$ \\
RMS input voltage, $u_{c_{r m s}}$ & $\leq 300$ & $32-108$ & $63-80$ & $V$ \\
Airgap peak, $\left(z_{t}-z\right)_{p}$ & $\leq 7.5$ & $5-7.5$ & $2.5-2.6$ & $m m$ \\
Input voltage, $u_{c_{p}}$ & $\leq 300$ & $37-53$ & $\approx 20$ & $V$ \\
Settling time, $t_{s}$ & $\leq 3$ & $\checkmark$ & $\checkmark$ & $s$ \\
Airgap steady state error, $e_{\left(z_{t}-z\right)_{s s}}$ & $=0$ & $\checkmark$ & $\checkmark$ & $m m$ \\
Phase margin,PM & $35^{\circ}-45^{\circ}$ & $35^{\circ}-45^{\circ}$ & $\mathbf{3 . 3}^{\mathbf{o}}-\mathbf{5 . 5}$ & \\
Outer bandwidth, $f_{b_{\text {out }}}$ & $\leq 20$ & $2.9-3.2$ & $\approx 4$ & $\mathrm{~Hz}$ \\
Inner bandwidth, $f_{b_{\text {in }}}$ & $50-100$ & $70-100$ & $\approx 100$ & $\mathrm{~Hz}$ \\
\hline \hline
\end{tabular}

tuned controllers appears (i.e. those that comprise the Pareto-optimality between the objective functions). Therefore a large number of controllers exists when thee sensor optimisation framework is executed. That is a function of the number of chromosomes in the population and is given as

$$
N_{K}=N_{s} \times n_{c}
$$

where $N_{K}$ is the total number of controllers at the completion of the framework, $N_{s}$ is the number of feasible sensor sets and $n_{c}$ is the number of chromosomes in the population. Thus, an indication is needed to distinguish which controllers on the Pareto front of the corresponding sensor set satisfy the control constraints. This can be done using (12) and it can be demonstrated via two examples using the Pareto fronts of $\left\{\left(z_{t}-z\right), b\right\}$ and $\left\{\left(z_{t}-z\right), i\right\}$. A pair of controllers that result to the best ride quality (i.e minimum vertical acceleration), is selected for each sensor set. The parameters that form the controllers in (14) are tabulated in Table 5. The

\begin{tabular}{cccccc} 
Table 5. & Selected controllers' parameters from $\left\{\left(z_{t}-z\right), i\right\}$ and $\left\{\left(z_{t}-z\right), b\right\}$. \\
\hline \hline & $G_{P I}$ & $\tau_{i}$ & $G_{P A}$ & $\tau_{o}$ & $k_{o}$ \\
\hline$\left\{\left(z_{t}-z\right), b\right\}$ & $11949 \mathrm{~V} / \mathrm{T}$ & $0.0181 \mathrm{~s}$ & $3.547 \mathrm{~T} / \mathrm{m}$ & $0.038 \mathrm{~s}$ & 3.92 \\
$\left\{\left(z_{t}-z\right), i\right\}$ & $237 \mathrm{~V} / \mathrm{A}$ & $0.023 \mathrm{~s}$ & $790.5 \mathrm{~A} / \mathrm{m}$ & $0.00321 \mathrm{~s}$ & 2.493 \\
\hline \hline
\end{tabular}


resulting closed-loop responses of the suspension for each sensor set are shown on Table 6 . For the $\left\{\left(z_{t}-z\right), b\right\}$ configuration the penalty values are zero since all constraints are satisfied in contrast to $\left\{\left(z_{t}-z\right), i\right\}$ where the ride quality and the phase margin (PM) are violated. Consequently, the overall constraint violation function $\Omega$, is zero for $\left\{\left(z_{t}-z\right), b\right\}$ but 1.077 for $\left\{\left(z_{t}-z\right), i\right\}$. In that sense, using the $\Omega$ it is possible to distinguish which controllers satisfy the control constrains in every feasible sensor set and additionally, indicate which sensor set can be used for control with satisfactory performance.

\begin{tabular}{|c|c|c|c|c|}
\hline & \multicolumn{2}{|c|}{$\overline{\left\{\left(z_{t}-z\right), b\right\}}$} & \multicolumn{2}{|c|}{$\overline{\left\{\left(z_{t}-z\right), i\right\}}$} \\
\hline Quantity & Actual value & Penalty value & Actual value & Penalty value \\
\hline$\ddot{z}_{r m s}$ & $0.260 \mathrm{~ms}^{-2}$ & 0 & $0.588 \mathrm{~ms}^{-2}$ & 0.174 \\
\hline$\left(z_{t}-z\right)_{r m s}$ & $1.5 \mathrm{~mm}$ & 0 & $1.76 \mathrm{~mm}$ & 0 \\
\hline$u_{c_{r m s}}$ & $32.4 \mathrm{~V}$ & 0 & $63.14 \mathrm{~V}$ & 0 \\
\hline$\left(z_{t}-z\right)_{p}$ & $7.48 \mathrm{~mm}$ & 0 & $2.6 \mathrm{~mm}$ & 0 \\
\hline$u_{c_{p}}$ & $53.6 \mathrm{~V}$ & 0 & $20.53 \mathrm{~V}$ & 0 \\
\hline$t_{s}$ & $2.37 \mathrm{~s}$ & 0 & $2.38 \mathrm{~s}$ & 0 \\
\hline$e_{\left(z_{t}-z\right)_{s s}}$ & $6.39 \times 10^{-6} \mathrm{~m}$ & 0 & $1 \times 10^{-6} \mathrm{~m}$ & 0 \\
\hline $\mathrm{PM}$ & $35^{\circ}$ & 0 & $3.38^{\circ}$ & 0.903 \\
\hline$f_{b_{\text {out }}}$ & $3.06 \mathrm{~Hz}$ & 0 & $4.01 \mathrm{~Hz}$ & 0 \\
\hline$f_{b_{i n}}$ & $98 \mathrm{~Hz}$ & 0 & $98.87 \mathrm{~Hz}$ & 0 \\
\hline$\Omega$ & & 0 & & 1.077 \\
\hline
\end{tabular}

\section{LQG Control of the EMS system within the framework}

In this Section, a short description of the LQG controller formulation for the EMS system as it is merged into the proposed framework is done. The LQG controller design is performed according to the separation principle, as described by Skogestad and Postlethwaite (2005). The state feedback gains (LQR design), $-K_{l q r}$, are appropriately selected in order to achieve the desirable control properties while the KBE is merged into the loop at the second stage, to provide appropriate state estimation. The LQG control problem is to find the optimal control $u$ which minimizes the following performance index in (16). Note that output regulation is considered rather than state regulation. This index has to be solved for every feasible sensor set of the EMS system.

$$
J=E\left\{\lim _{T \rightarrow \infty} \int_{0}^{T}\left[y^{T} Q y+u^{T} R u\right] d t\right\}
$$

\subsection{LQR control for the suspension}

A linear quadratic feedback controller can be used to control the non-linear EMS model as illustrated in Fig. 6. An extra state is selected forming a Proportional plus Integral (P+I) state regulator. The integral action on airgap is an extra state forming a four-state vector given as $x=\left[i, \dot{z},\left(z_{t}-z\right), \int z_{t}-z\right]^{T}$. The aim of the particular LQR design is to appropriately select a state feedback vector, $-K_{l q r}$ which minimizes the performance index in (17). The performance index represents output regulation of the EMS system with integral action on the airgap.

$$
J=\int_{0}^{T}\left[y^{T} Q_{o} y+u^{T} R u\right] d t
$$

where $y=\left[\ddot{z}\left(z_{t}-z\right) \int\left(z_{t}-z\right)\right]^{T}$ and $u=u_{c}$. $Q_{o}(3,3)$ regulates the speed of response $\int\left(z_{t}-z\right)$ while $Q_{o}(1,1)$ and $Q_{o}(2,2)$ regulates the vertical acceleration and the airgap respectively. 


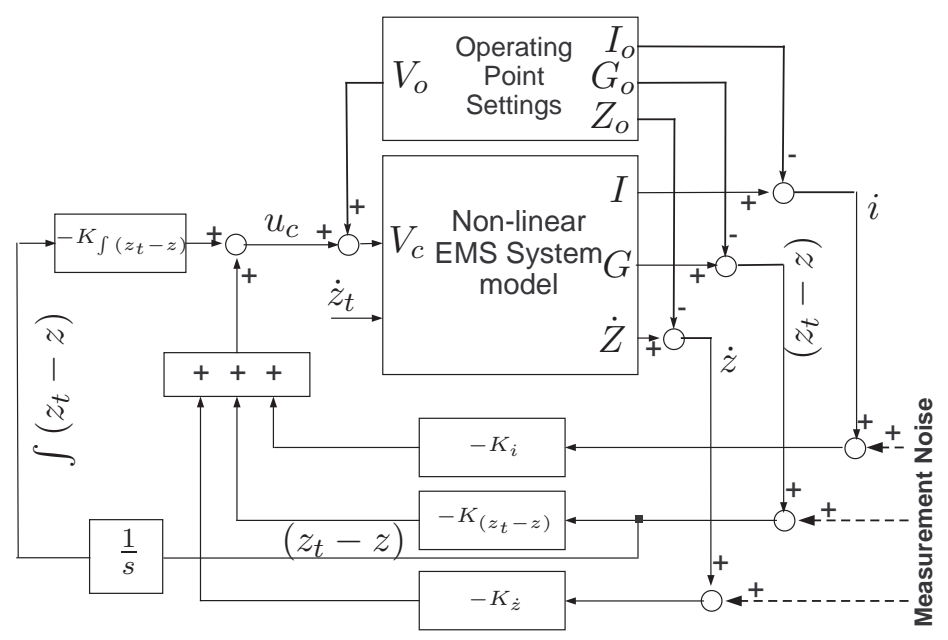

Figure 6. LQR control for the nonlinear EMS system.

The output regulation weighting matrices are based on Bryson's rule (Franklin et al. 2002). $Q_{o}$ is given in diagonal form in (18) while the input weighting matrix $R$ is a scalar value because the EMS is a single input system.

$$
Q_{o}=\operatorname{diag}\left(\frac{1}{q_{\ddot{z}}^{2}}, \frac{1}{q_{\left(z_{t}-z\right)}^{2}}, \frac{1}{q_{\int\left(z_{t}-z\right)}^{2}}\right), R=1 / r^{2}
$$

The tuning of the $Q_{o}$ and $R$ aim to minimize the EMS response on straight track irregularities (i.e. minimize the vertical acceleration and coil current) while enhancing the performance onto gradient track move. NSGA-II is used for optimal tuning of $Q_{o}$ and $R$. The selected NSGAII parameters for the LQR optimisation are given on Table 3 . Four variables $\left(n_{V}=4\right)$ with real-coded values are used to represent the output weight factors in (18).

\subsection{The Kalman-Bucy estimator design}

The linear time-invariant KBE has a state space form formally written as

$$
\begin{aligned}
& \dot{\hat{x}}=A \hat{x}+B_{u} u+K_{l q g}(y-\hat{y}) \stackrel{(\hat{y}=C \hat{x})}{=} A \hat{x}+B_{u} u+K_{l q g}(y-C \hat{x}) \\
& \hat{y}=C \hat{x}
\end{aligned}
$$

where $K_{l q g}$ is the observer gain matrix that minimizes $E\left\{[x-\hat{x}]^{T}[x-\hat{x}]\right\}$. Minimization can be achieved by appropriately tuning of the measurement noise covariance matrix $V$, and process noise covariance matrix $W$. The measurement noise weighting $(V)$ is given in $(21)$ for all five measurements. In a practical situation, this can be calculated from sensor equipment data sheets or prior simulation of baseline controller designs. In this case is not possible to have that information therefore it is assumed that the noise covariance for each corresponding measurement is equal to $1 \%$ of the peak value from each measurement of the deterministic track profile closed-loop response (this is something usual in the area of railway).

$$
V=\operatorname{diag}\left(V_{i}, V_{b}, V_{\left(z_{t}-z\right)}, V_{\dot{z}}, V_{\ddot{z}}\right)
$$

In this design the process noise matrix $B_{w}=B_{\dot{z}_{t}}$ and the process noise covariance matrix $W$ refers to the track velocity input and this is optimally tuned for each sensor set using the NSGA- 
II (this is a realistic process noise input to the system).

The LQG control for the EMS is illustrated with the block diagram in Fig. 7. During the execution of the framework algorithm the errors between the estimated and the 'ideal' states are minimized i.e. the comparison between the closed-loop response with the LQR and the response with the KBE in the loop. The minimization of the errors is performed by the NSGA-II for each one of the sensor sets. For this purpose, the objective functions to be minimized are: (i)

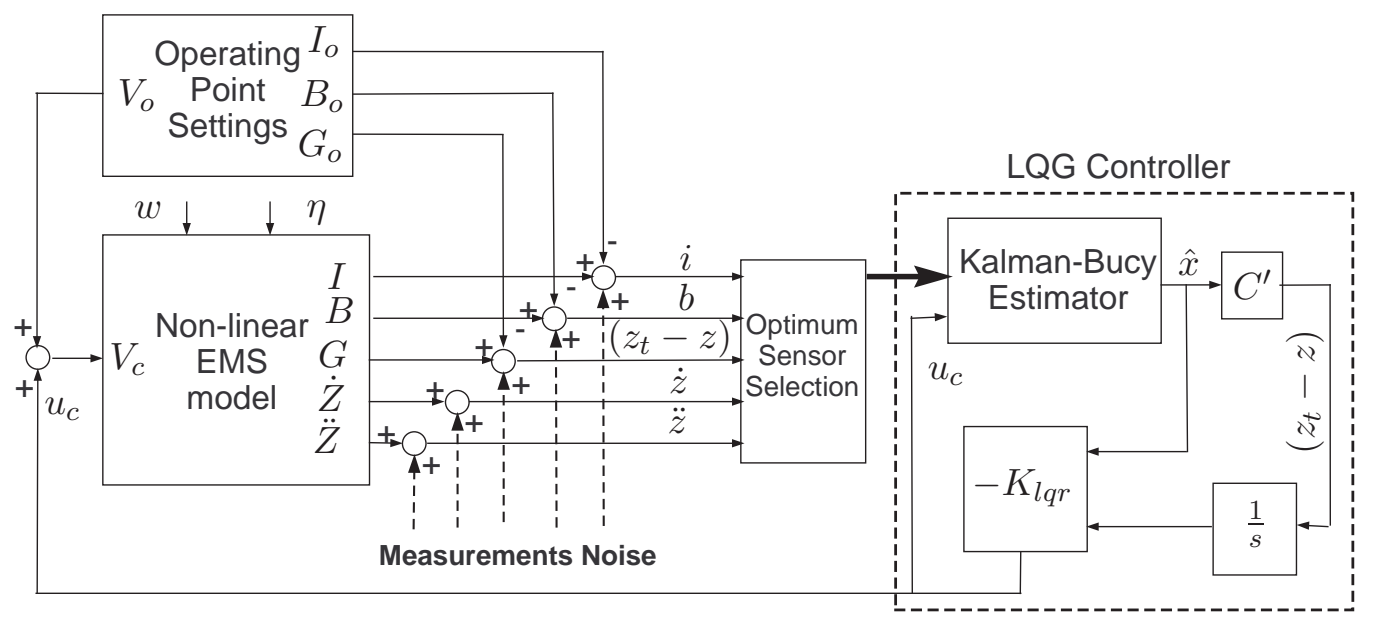

Figure 7. LQG control with optimum sensor selection for the non-linear EMS system

the Integral Absolute Error (IAE) for the closed-loop response with deterministic disturbance expressed in (22) and (ii) the Root Mean Square Error (RMSE) for the stochastic closed-loop response described in (23) (Panagopoulos et al. 2002). An extra objective function is added (24) which is the RMS value of the noise that appears on the input voltage $\left(u_{c}\right)$ from the measurements of the suspension with idle track profile. The final objective functions for the LQG controller tuning are formally written as

$$
\begin{aligned}
& \phi_{d_{1,2,3}}=\int_{0}^{t}\left|x_{o}-x_{a}\right| d t \\
& \phi_{s_{4,5,6}}=R M S\left(x_{o}-x_{a}\right) \\
& \phi_{7}=u_{n_{r m s}}
\end{aligned}
$$

This makes a total of 7 individual objective functions (minimized for each sensor set) where $x_{o}$ is the vector of the monitored states of interest of the closed-loop with the LQR state feedback (e.g. 'ideal' closed-loop response) and $x_{a}$ the monitored states of interest of the closed-loop with the overall LQG controller, e.g. actual closed-loop (prior to adding sensor noise). Note that the EMS system is open-loop unstable and this assessment in tuning is quite attractive i.e taking directly into account the domain differences.

After the optimal tuning of the LQG controller for each sensor set, there is a large number of controllers (equal to the population number). Hence the overall penalty parameter $\Omega$ in (12), is used to assist with the best controller selection as it shown in the flow chart of Fig. 2. Since for a sensor set there could be (which is the usual case) more that one controllers in the population that satisfy all control constraints another criterion is needed to select the best one among those. This is given as the sum of the objective functions in (22) and (23) (states error for deterministic and stochastic responses with and without the KBE) as shown below

$$
S_{f}=\sum_{i=1}^{n_{x}} \phi_{d_{i}}+\sum_{j=1}^{n_{x}} \phi_{s_{j}}
$$


where $n_{x}$ is the number of estimated states $\left(n_{x}=3\right)$.

In that way it is ensured that the selected controller for the corresponding sensor set satisfies the control constraints and has accurate state estimation.

\section{Simulations and data analysis}

In this Section the efficacy of the proposed framework is assessed using the EMS system. The framework algorithm is executed in two parts. The first part is the LQR tuning where the selection of the 'ideal' closed-loop response is done. Throughout the second part (where the sensor information becomes critical) KBE is optimally tuned for every feasible sensor set i.e. observability is tested for every sensor set.

Figure 8 shows the Pareto-Optimality between the objectives in (9) with the control constraints listed on Table 2 being satisfied. Figure 9 depicts the airgap $\left(z_{t}-z\right)$ and input voltage $\left(u_{c}\right)$ deviations for the deterministic closed-loop response using the 50 optimally tuned controllers that comprise the last generation of the LQR tuning. It is illustrated that the control constraints for the deterministic response of thee EMS are restricted to the values as listed on Table 2 (note that for good resolution the noise is removed form the measurements). At this point, the LQR

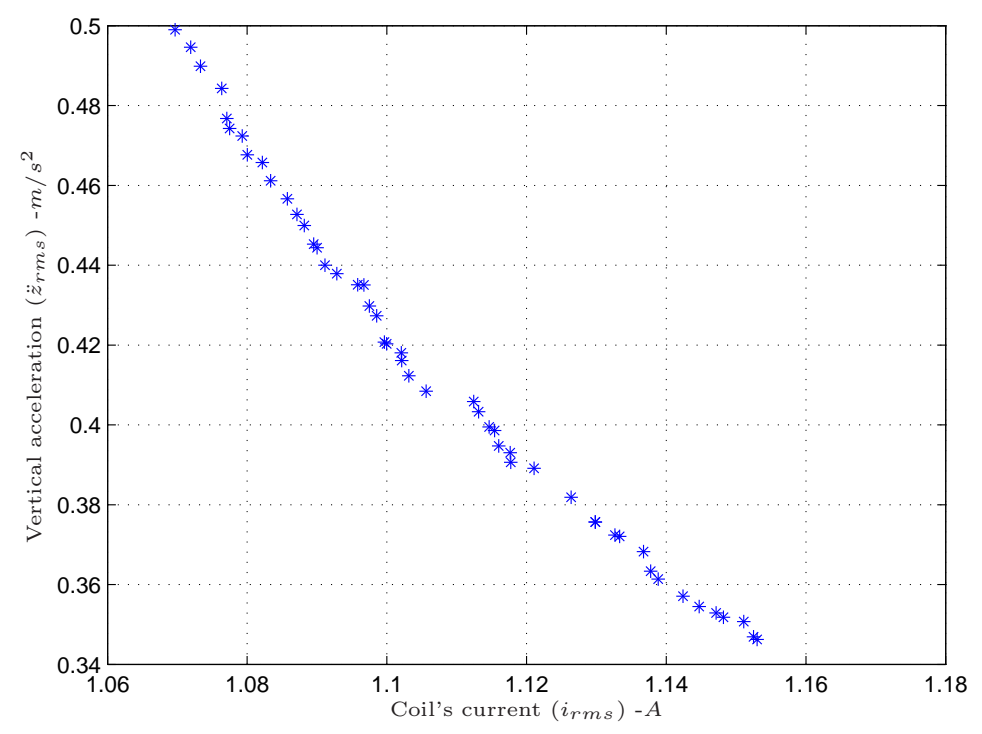

Figure 8. Pareto-optimality from the tuning of LQR control.

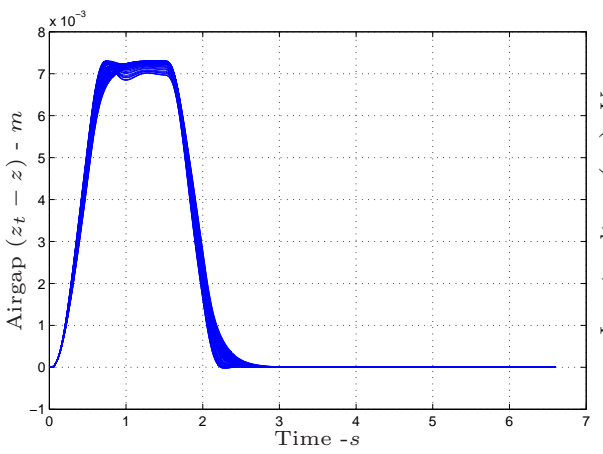

(a) Airgap deviations

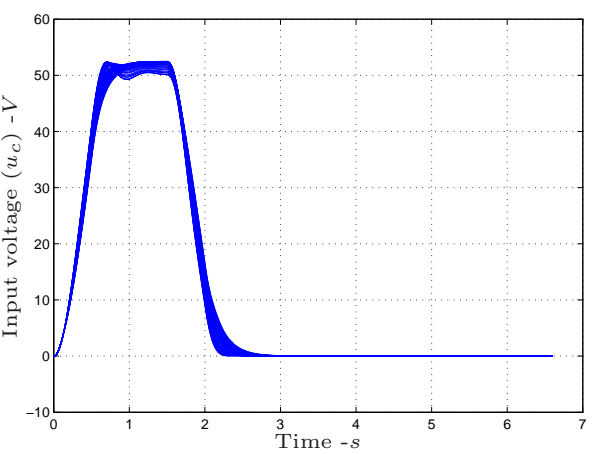

(b) Input voltage deviations

Figure 9. Deterministic closed-loop responses of the EMS system with the 50 optimally tuned state feedback controllers. 
state gain vector $K_{l q r}$, that results in the 'ideal' closed-loop performance can be selected. The choice of the desired closed-loop response is based on the user's controller selection criteria $\left(f_{c_{i}}, f_{u}\right)$ as follows

(1) Guarantee that the initially selected controllers result to the closed-loop response with vertical acceleration of less than $0.5 \mathrm{~m} / \mathrm{s}^{2} \cdot\left(f_{c_{1}} \equiv \ddot{z}_{r m s}<0.5 \mathrm{~m} / \mathrm{s}^{2}\right)$.

(2) and ensure that the excitation coil's current from the closed-loop response of less than $2 \mathrm{~A}$ $\left(f_{c_{2}} \equiv i_{r m s}<2 A\right)$.

(3) Ensure that the finally selected state gain vector $K_{l q r}$, results to the best ride quality $\left(f_{u} \equiv\right.$ $\left.\min \left(\ddot{z}_{r m s}\right)\right)$.

The closed-loop response with the selected gains results in an RMS vertical acceleration of $0.346 \mathrm{~m} / \mathrm{s}^{2}$ with the corresponding state gains tabulated on Table 7 . The resulting performance with these gains is depicted in Fig. 10.

The second part of the framework is the optimisation of the KBE for each sensor set. The aim

Table 7. The selected optimum $\mathrm{LQR}$ gain vector values
\begin{tabular}{cccc}
\hline \hline$K_{i}$ & $K_{\dot{z}}$ & $K_{\left(z_{t}-z\right)}$ & $K_{\int\left(z_{t}-z\right)}$ \\
\hline $\mathrm{V} / \mathrm{A}$ & $\mathrm{V} /(\mathrm{m} / \mathrm{s})$ & $\mathrm{V} / \mathrm{m}$ & $\mathrm{V} / \mathrm{m}$ \\
\hline-246.85 & $-3.366 \times 10^{3}$ & $2.145 \times 10^{5}$ & $2.417 \times 10^{5}$ \\
\hline
\end{tabular}

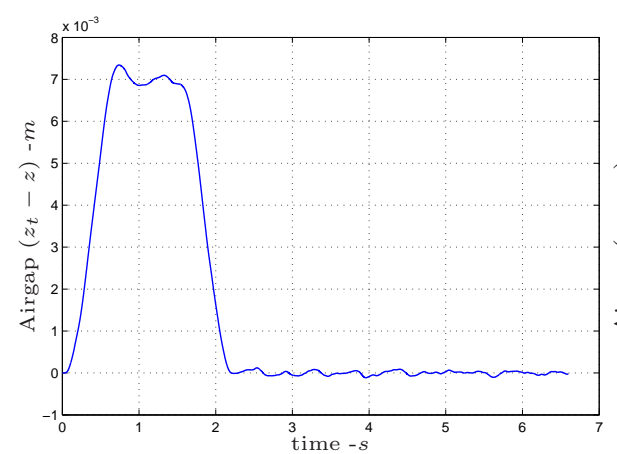

(a) Airgap response for deterministic behaviour of the track.

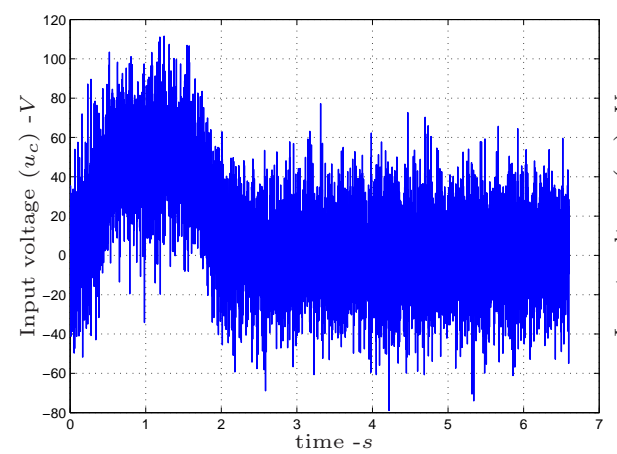

(c) Input voltage for deterministic behaviour of the track.

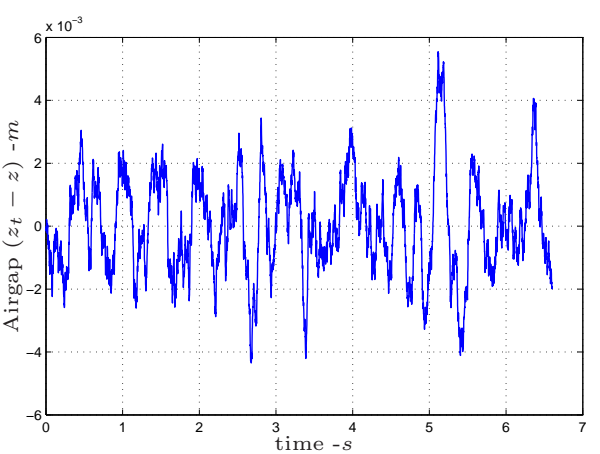

(b) Airgap response for stochastic behaviour of the track.

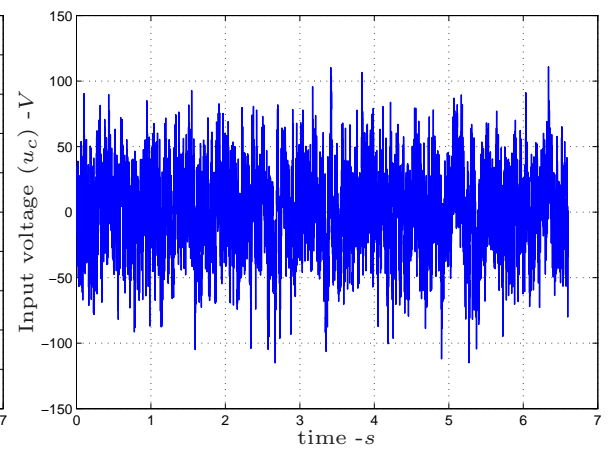

(d) Input voltage for stochastic behaviour of the track.

Figure 10. 'Ideal' closed-loop response of the suspension with LQR control.

is to achieve the same closed-loop response for each sensor set as given in Fig. 10. According to (15) and since all sensor sets are feasible at the completion of the framework, there are 620 optimally tuned KBEs. By appropriate controller selection for each sensor set using (12) and (25) the end result gives an optimally tuned KBE for each sensor set. There are 24 out of 31 sensor sets found to give the same closed-loop response as the 'ideal'. 
The results listed on Table 8 include some of the optimally tuned sensor sets. The values of the third row correspond to the 'ideal' closed-loop response. The second column lists the sensor sets and the first column the corresponding identification number. Columns $3-5$ show the values for the closed-loop response from the stochastic track profile and columns $6-9$ show the values from the closed-loop response from the deterministic track profile. $10^{\text {th }}$ column lists the level of the noise on the control input taken with the idle track profile. Note the reduction of the level of the noise with the LQG controller compared to the LQR response. The last column indicates whether the corresponding sensor set satisfies the control constraints $(\checkmark)$ or not (x).

\begin{tabular}{|c|c|c|c|c|c|c|c|c|c|c|}
\hline \multirow[t]{2}{*}{ id } & \multirow{2}{*}{$\begin{array}{c}\text { Sensor } \\
\text { Set }\end{array}$} & $\left(z_{t}-z\right)_{r m s}$ & $u_{c_{r m s}}$ & $\ddot{z}_{r m s}$ & $\left(z_{t}-z\right)_{p}$ & $u_{c_{p}}$ & $t_{s}$ & $e_{\left(z_{t}-z\right)_{s s}}$ & $u_{n_{r m s}}$ & $\Omega$ \\
\hline & & $\mathrm{mm}$ & $\mathrm{V}$ & $m s^{-2}$ & $\mathrm{~mm}$ & $\mathrm{~V}$ & $s$ & $\mathrm{~mm}$ & $\mathrm{~V}$ & \\
\hline & LQR response $\rightarrow$ & 1.5 & 22.64 & 0.34 & 7.3 & 52.4 & 2.16 & 0.019 & 25.9 & $\checkmark$ \\
\hline 1 & $i$ & 1.78 & 29.16 & 0.50 & 2.09 & 22.93 & 6.18 & 0.18 & 2.95 & $\mathrm{x}$ \\
\hline 2 & $b$ & 1.46 & 22.47 & 0.32 & 6.74 & 63.82 & 2.18 & 0.019 & 5.81 & $\checkmark$ \\
\hline 3 & $\left(z_{t}-z\right)$ & 1.49 & 22.41 & 0.31 & 10.69 & 84.83 & 2.56 & 0.77 & 4.84 & $\mathrm{x}$ \\
\hline 4 & $\dot{z}$ & 1.57 & 171 & 6.83 & 14.6 & 709 & 6.58 & 8.78 & 210 & $\mathrm{x}$ \\
\hline 5 & $\ddot{z}$ & 1.46 & 22.44 & 0.32 & 6.82 & 63.04 & 2.19 & 0.013 & 5.89 & $\checkmark$ \\
\hline 6 & $i, b$ & 1.42 & 22.24 & 0.31 & 6.73 & 59.13 & 2.18 & 0.024 & 4.03 & $\checkmark$ \\
\hline 7 & $i,\left(z_{t}-z\right)$ & 1.45 & 22.25 & 0.31 & 10.81 & 85.21 & 2.77 & 0.76 & 3.69 & $\mathrm{x}$ \\
\hline 8 & $i, \ddot{z}$ & 1.46 & 22.18 & 0.32 & 6.82 & 58.91 & 2.18 & 0.03 & 4.00 & $\checkmark$ \\
\hline 9 & $b,\left(z_{t}-z\right)$ & 1.43 & 22.17 & 0.32 & 6.81 & 57.41 & 2.18 & 0.027 & 3.69 & $\checkmark$ \\
\hline 10 & $b, \ddot{z}$ & 1.43 & 22.20 & 0.32 & 6.78 & 59.64 & 2.18 & 0.011 & 4.11 & $\checkmark$ \\
\hline 11 & $i, b,\left(z_{t}-z\right)$ & 1.46 & 22.06 & 0.32 & 6.79 & 55.99 & 2.18 & 0.02 & 3.08 & $\checkmark$ \\
\hline 12 & $i, b, \dot{z}$ & 1.46 & 22.21 & 0.32 & 6.79 & 59.55 & 2.20 & 0.06 & 4.03 & $\checkmark$ \\
\hline 13 & $i, b, \ddot{z}$ & 1.42 & 22.24 & 0.31 & 6.80 & 59.55 & 2.20 & 0.06 & 3.29 & $\checkmark$ \\
\hline 14 & $i,\left(z_{t}-z\right), \dot{z}$ & 1.48 & 22.18 & 0.32 & 7.69 & 63.04 & 2.35 & 0.10 & 3.69 & $\mathrm{x}$ \\
\hline 15 & $i, b, \dot{z}, \ddot{z}$ & 1.42 & 22.11 & 0.32 & 6.81 & 56.82 & 2.19 & 0.03 & 3.29 & $\checkmark$ \\
\hline 16 & $i,\left(z_{t}-z\right), \dot{z}, \ddot{z}$ & 1.43 & 22.05 & 0.32 & 6.90 & 58.55 & 2.18 & 0.03 & 3.07 & $\checkmark$ \\
\hline 17 & $i, b,\left(z_{t}-z\right), \dot{z}, \ddot{z}$ & 1.46 & 22.02 & 0.32 & 6.84 & 55.98 & 2.19 & 0.03 & 2.70 & $\checkmark$ \\
\hline
\end{tabular}

\subsection{Optimum sensor selection for control}

An initial performance comparison between the 'ideal' response and the LQG responses with each of the sensor sets shows that the performance doesn't change significantly. Although there is some small variation on the performance with the LQG most of sensor sets can be used to control the suspension. Since at this stage appropriate control of the suspension is the main task, one may conclude that instead of using 5 sensors (id:17), only one can be used (id:2 or id:5). The flux density (id:2) as well as vertical acceleration (id:5) measurements are good choices ensuring that the suspension performance is adequate. A single sensor is a good first choice to provide an insight into performance issues and simplified solutions. In this context, Fig. 11 illustrates the state estimation using a single sensor i.e $\mathcal{Y}_{\text {opt }}=\mathrm{id}: 5$. This choice provides a rather appropriate set of state estimates, with a small error of the estimation of the vertical velocity state. However, the performance of the suspension is not affected. The response of the suspension with id:5 is illustrated in Fig. 12 where the deterministic closed-loop response of the suspension is reflected on the airgap (Fig. 12(a)) and the input voltage (Fig. 12(b)). Both airgap and input voltage are within the control constraints given in Section 3.3.

\subsection{Optimum sensor selection for fault tolerant control}

The EMS system is a safety-critical system hence the sensor fault tolerance is a vital issue and should be carefully considered in control design. At this point, it worth noting the importance of the results from the proposed framework regarding the sensor fault tolerance. In particular, a longer set of sensors provides more information to the controller, and monitors more signals. Thus provides the means of switching to different controllers (with subsets of sensors), if necessary, subject to sensor fault conditions and controller banks ready from the off-line framework for maintaining performance (or almost the 'ideal' performance). However, longer set of sensors could 

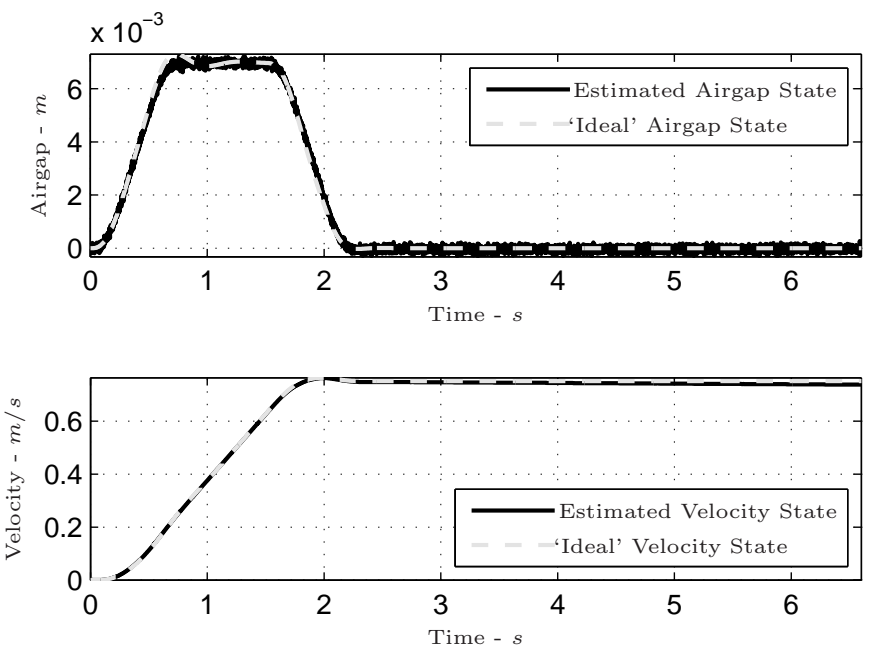

Estimated current state and Ideal current state

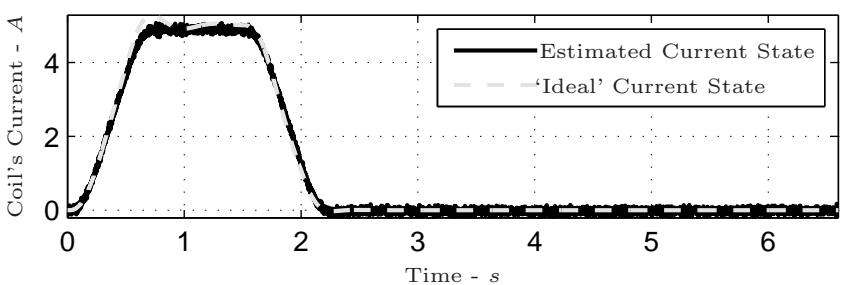

Figure 11. The estimated and the 'ideal' states using id:5.

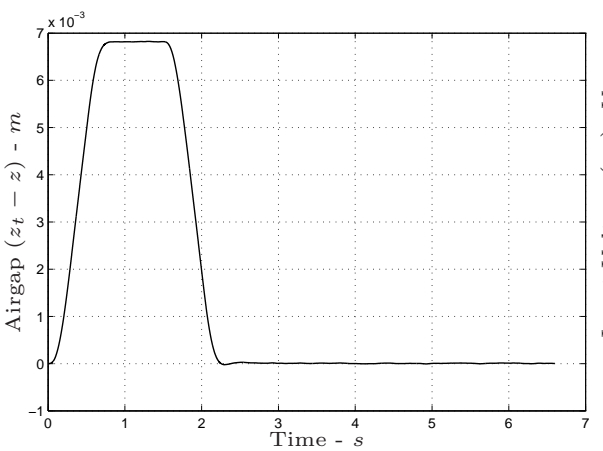

(a) Airgap deviation $\left(z_{t}-z\right)$.

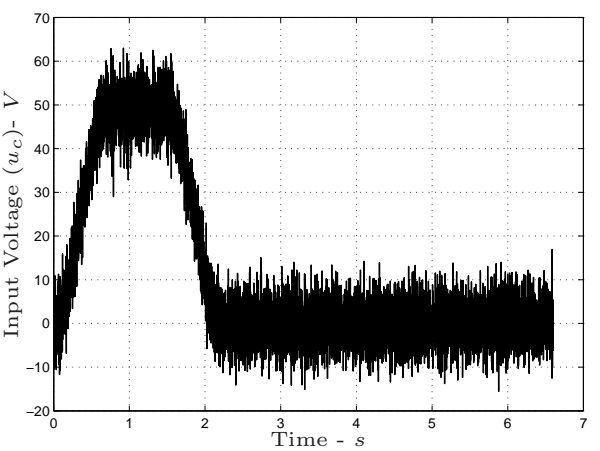

(b) Input voltage $\left(u_{c}\right)$.

Figure 12. EMS system closed-loop response to deterministic track profile using id:5.

imply increased number of sensor fault probability. On the contrary, a single sensor provides simple straight forward hardware redundancy (by using duplicates of the sensor in a voting selection scheme) but limited costs (in the case of hardware redundancy). Clearly, the information from the sensor optimisation framework can be used in order to apply FTC for multiple sensor failures, minimizing the sensor redundancy and maintain optimum performance under relevant sensor fault conditions as illustrated in Fig. 13 (Blanke et al. 2003). When one or more sensors fail remedial actions are taken via controller reconfiguration and the performance is restored. At this point it is possible to answer the question that arose from Section 2 using the simulation results from the framework listed on Table 8. Aiming to cover multiple sensor faults using id:17, i.e. the full sensor set, a bank of $\mathrm{KBE}$ can be used in order to restore performance following multiple sensor faults. In fact, when sensor faults happen the performance of the suspension is easily predicted from the responses of the remaining healthy sensors listed (assuming that 


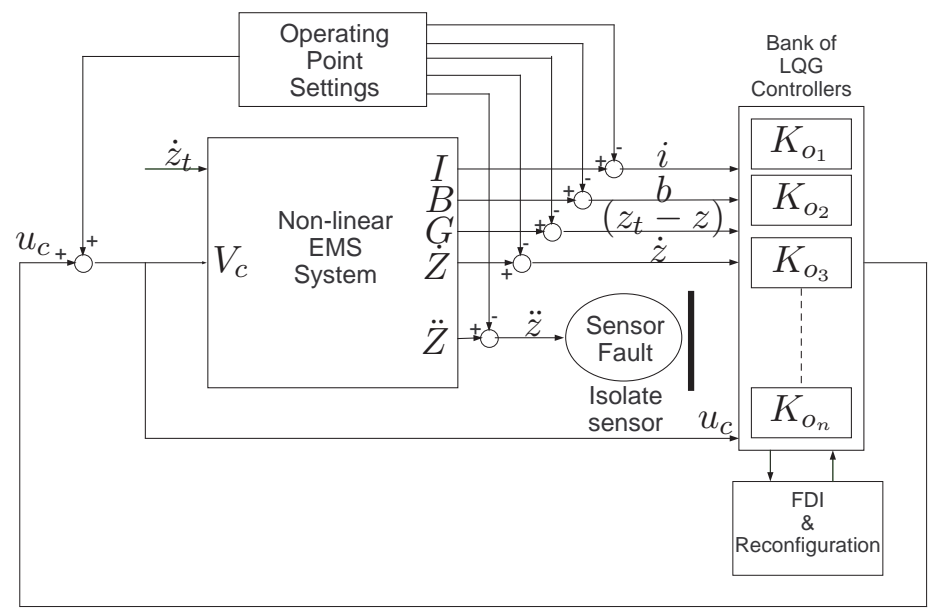

Figure 13. The proposed FTC diagram for multiple sensor failures.

stability is maintained during controller reconfiguration). For example assume that id:17 is used for the control of the suspension. If four sensors fail, the only remain healthy sensor will be the airgap. With that fault scenario in mind it is possible to lead to catastrophic failure of the suspension because there is a serious constraint violation on the airgap maximum allowed deviation $\left(\left(z_{t}-z\right)=10.69 \mathrm{~mm}\right.$ in row id:3). Under this scope the airgap sensor must never remain alone. This problem can be overcome by perhaps using redundant sensors with the other sensors in id:17. Nevertheless, different sensor fault conditions can lead to id:4, id:7 or id:14 that result to serious airgap constraint violation and then the problem becomes complex since a lot of redundant sensors are necessary in id:17 in order to cover all sensor fault scenarios.

The alternative approach is to avoid using the full sensor set. A second option is id:11 with current, flux and airgap measurements. The problem with this sensor set is that only four sensor fault conditions can be covered via controller reconfiguration (i.e id:1, id:2, id:6 and id:9) and the remaining two i.e id:3 and id:7, result to critical constraint violation (airgap). Another drawback is that the airgap sensor is generally expensive and vulnerable to external hazards. A different option is id:12 with current, flux and velocity measurements. Again, with this sensor set the performance can be covered using only three out of six fault conditions with id:1, id:2 and id:6. A sensor set with four sensors or the full sensor set could be selected but in such case more sensor fault probabilities are introduced with increasing number of sensors (i.e four sensors equals to $2^{4}-1=15$ sensor fault conditions).

Starting with a different reason, assume that the worst sensor fault condition is to remain with one sensor. Examining the single sensors it can be seen that id:3 and id:4 have serious constraint violation (i.e. airgap). The performance with id:1 is acceptable with a longer settling time and both id:2 and id:5 have acceptable performance. From this point of view, the id:13, that includes id:1,id:2 and id:5, can be used for controlling the EMS. Note that id:13 and id:17 have very similar performance, therefore if id:13 is used, the worst resulting performance comes when both $b$ and $\ddot{z}$ fail i.e. the response with id: 1 which has long settling time and a small steady state error. With the sensor set id:13, there are six possible sensor fault conditions that could happen. The first three are individual faults on current, flux and acceleration sensors and the remaining three are combined current/flux, current/acceleration and acceleration/flux sensor faults. Note that here simultaneous faults are taken into account that is the worst case for two sensor faults. As explained, in order to accommodate the 6 possible fault conditions via controller reconfiguration a bank of 6 KBEs can be used. Under this scope, the best sensor set that can be used for control and fault tolerance is $\mathcal{Y}_{\text {opt }}=\mathrm{id}: 13$. 


\subsubsection{Sensor fault tolerance test}

In order to test the sensor set with id:13 for sensor fault tolerance some sensor fault scenarios will be considered. As Isermann (1997) indicates faults are distinguished in three main types: Abrupt, Incipient and Indeterminant faults. The former type is used for the fault(s) scenario(s): at time $=1 s$ the sensor(s) is(are) impaired giving wrong readings with random low frequency characteristics. The fault profile for the accelerometer is illustrated in Fig. 14 for both deterministic and stochastic closed-loop responses. Similar fault profile is used for the other two sensors and for the combination of faults the same profiles are used accordingly.

When faults occur the AFTC scheme should take remedial actions in order to maintain the

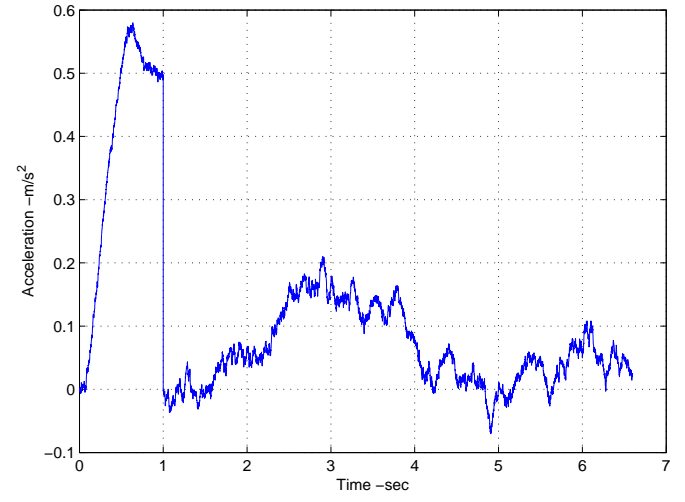

(a) Faulty acceleration measurement for the deterministic response.

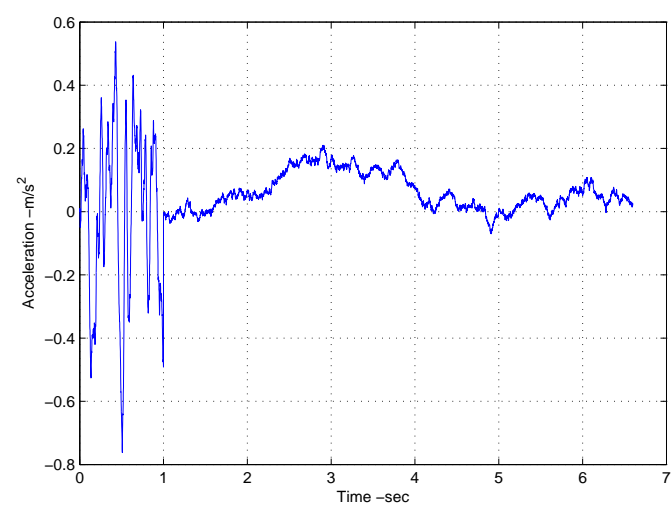

(b) Faulty acceleration measurement for the stochastic response.

Figure 14. Fault profiles for the accelerometer sensor (fault at 1 second).

performance of the EMS system. Particularly, for the accommodation of the sensor faults a bank of KBEs is used where appropriate reconfiguration is done. For this purpose a Fault Detection and Isolation mechanism is needed. The Fault Detection (FD) mechanism is used to detect the faulty sensors and the isolation unit is used to remove them from the loop (i.e. using individual switches). Model-based FD has been implemented in many practical applications (Isermann and Ball 1997). A typical way to detect fault(s) is by monitoring the corresponding residual(s) of the desired components (i.e. actuators, sensors). Various methods exist for the residual generation including the dedicated observer method, the parity space, FD filter and parameter identification approaches (Isermann 1997, Frank 1990). When KBE are used for control, a common approach for the fault detection is to use the residual $r_{y_{i}}$ (where $y_{i}$ is the corresponding sensor).

The controller reconfiguration is achieved using a binary vector that is residual dependent and indicates that state of each sensor in the set i.e. which and if a sensor is healthy or faulty (Blanke et al. 2003). Faulty sensors are indicated by ' 1 ' and healthy by ' 0 '. For example, if $i, b, \ddot{z}$ results to the binary vector $\left[\begin{array}{lll}0 & 0 & 0\end{array}\right]$ means that all measurements are healthy and the corresponding $\mathrm{KBE}$ is used i.e $K_{o_{i, b, \ddot{z}}}$. If the residual of the current sensor is larger than a predefined threshold

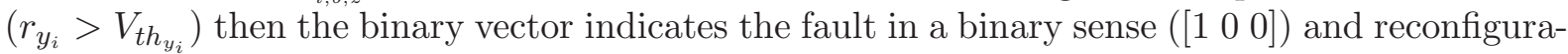
tion takes place by switching to the relevant KBE i.e. $K_{o_{b, z}}$. The possible sensor fault conditions with the resulting binary vector and the corresponding KBE are listed on Table 9.

The next step is to test the sensor fault tolerance under different fault conditions (stability is tested and negligible switching time is assumed). By testing all 6 possible sensor fault conditions the stability and performance for the stochastic inputs are both maintained. The same tests are done for the deterministic input and found stability to be well recovered for all possible fault scenarios. Under deterministic inputs the performance is, in most cases, maintained except when faults happen simultaneously on the flux density and acceleration measurements at $1 s$. The problem is that the airgap deflection becomes very large during the transition from controller $K_{o_{i, b, z}}$ to $K_{o_{i}}$. However, extra hardware redundancy can be used for $b$ and/or $\ddot{z}$ in order 


\begin{tabular}{|c|c|c|c|c|c|}
\hline \multirow{2}{*}{$\begin{array}{l}\text { Healthy } \\
\text { Sensor(s) }\end{array}$} & \multirow{2}{*}{$\begin{array}{c}\text { Faulty } \\
\text { Sensor(s) }\end{array}$} & \multirow{2}{*}{$\begin{array}{c}\text { Binary } \\
\text { Vector }^{\text {a }}\end{array}$} & \multirow{2}{*}{$\begin{array}{c}\text { Kalman-Bucy } \\
\text { estimator }\end{array}$} & \multicolumn{2}{|c|}{ EMS Performance } \\
\hline & & & & \multirow{2}{*}{$\begin{array}{l}\text { Deterministic } \\
\text { Input }\end{array}$} & \multirow{2}{*}{$\begin{array}{l}\text { Stochastic } \\
\text { Input }\end{array}$} \\
\hline & & {$\left[\begin{array}{lll}i & b & \ddot{z}\end{array}\right]$} & & & \\
\hline$i, b, \ddot{z}$ & - & {$\left[\begin{array}{lll}0 & 0 & 0\end{array}\right]$} & $\overline{K_{o_{i, b}, \ddot{z}}}$ & & \\
\hline$i, b$ & $\ddot{z}$ & {$\left[\begin{array}{lll}0 & 0 & 1\end{array}\right]$} & $K_{o_{i, b}}$ & $\checkmark$ & $\checkmark$ \\
\hline$b, \ddot{z}$ & $i$ & {$\left[\begin{array}{lll}1 & 0 & 0\end{array}\right]$} & $K_{o_{b}, z}$ & $\checkmark$ & $\checkmark$ \\
\hline$i, \ddot{z}$ & $b$ & {$\left[\begin{array}{lll}0 & 1 & 0\end{array}\right]$} & $K_{o_{i, z}}$ & $\checkmark$ & $\checkmark$ \\
\hline$i$ & $b, \ddot{z}$ & {$\left[\begin{array}{lll}0 & 1 & 1\end{array}\right]$} & $K_{O_{i}}$ & $\mathrm{x}$ & $\checkmark$ \\
\hline$b$ & $i, \ddot{z}$ & {$\left[\begin{array}{lll}1 & 0 & 1\end{array}\right]$} & $K_{O_{b}}$ & $\checkmark$ & $\checkmark$ \\
\hline$\ddot{z}$ & $i, b$ & {$\left[\begin{array}{lll}1 & 1 & 0\end{array}\right]$} & $K_{o \ddot{z}}^{o}$ & $\checkmark$ & $\checkmark$ \\
\hline
\end{tabular}

a ' 0 '- Healthy sensor, ' 1 '- Faulty sensor

to avoid having the current sensor on its own (It is a scenario that really stretches the limits of the reconfiguration scheme but for maximum system safety can be considered).

The error between the airgap with fault-free condition $\left(z_{t}-z\right)$ and the airgap under the sensor fault condition $\left(z_{t}-z\right)_{f}$ is depicted in Fig. 15 under current and flux density faults at $1 s$. The stability is successfully maintained and the performance is well recovered as expected.

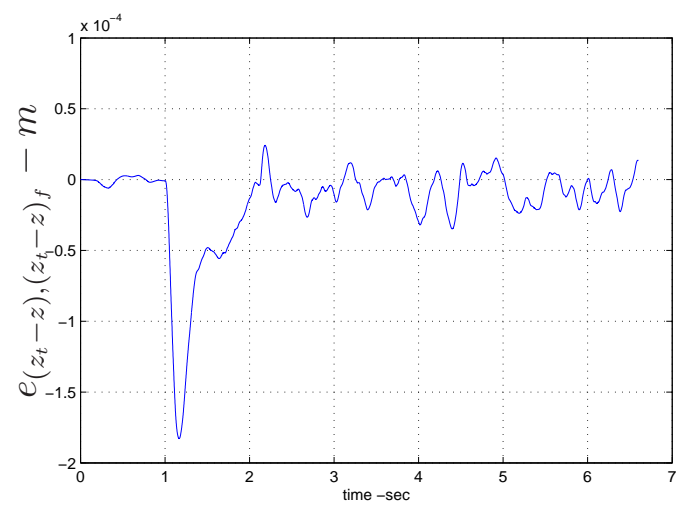

(a) Error between airgap with fault-free and faulty (b) Error between airgap with fault-free and faulty current/flux measurements $\left(e_{\left(z_{t}-z\right),\left(z_{t}-z\right)_{f}}\right)$ during current/flux measurements $\left(e_{\left(z_{t}-z\right),\left(z_{t}-z\right)_{f}}\right)$ during deterministic response.

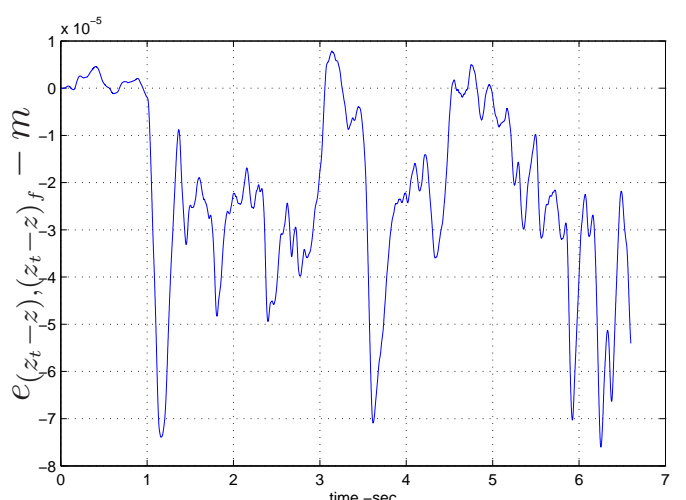

stochastic response.

Figure 15. Error between airgap with fault-free and faulty current/flux measurements.

\section{Conclusion}

The optimum sensor selection in control design is not a trivial task especially if the selection is performed subject to desirable multiple control system properties. In this paper the authors presented a systematic framework that simplifies sensor selection subject to system performance and reliability requirements. Without loss of generality the proposed framework is successfully implemented on a safety-critical system with non-trivial control requirements. Although time consuming (due to the fact that the performance has to be optimised for all feasible sensor sets) the framework is simple, flexible and can be used in a variety of practical engineering applications. The authors are currently investigating extensions of the framework to include robustness issues (as proposed by Dakev et al. (1997)), and of equipment cost encompassed in the objective functions.

\section{Acknowledgements}

Parts of this research were supported by Engineering and Physical Sciences Research Council, UK and BAE Systems (SEIC) under project Grand Ref. EP/D063965/1, and also by NEW-ACE EPSRC network under project ref. EP/E055877/1. In addition, the authors would like to thank 
the anonymous reviewers for their valuable comments and suggestions on improving the clarity of some parts of the paper.

\section{Notes}

(1) Throughout this paper, the signals taken from the closed-loop responses are with respect to the operating point indicated by the zero line on the graphs. For example the zero line for the airgap closed-loop response corresponds to the operating value of $15 \mathrm{~mm}$.

\section{References}

Anderson, B.D.O., and Moore, J.B., Optimal control: Linear quadratic methods, Upper Saddle River, NJ, USA: Prentice-Hall, Inc. (1990).

Blanke, M., Izadi-Zamanabadi, R., Bogh, S.A., and Lunau, C.P. (1997), "Fault-tolerant control systems - a holistic view," Control Engineering Practice, 5(5), 693-702.

Blanke, M., Kinnaert, M., Lunze, J., and Staroswiecki, M., Diagnosis and Fault-Tolerant Control, Secaucus, NJ, USA: Springer-Verlag New York, Inc. (2003).

Coello, C.A.C. (2002), "Theoretical and numerical constraint-handling techniques used with evolutionary algorithms: A survey of the state of the art," Computer Methods in Applied Mechanics and Engineering, 191(11-12), 1245-1287.

Dakev, N.V., Whidborne, J.F., Chipperfield, A.J., and Fleming, P.J. (1997), "Evolutionary $H_{\infty}$ design of an electromagnetic suspension control system for a maglev vehicle," Proceedings of the Institution of Mechanical Engineers.Part I, Journal of Systems $\& 3$ Control Engineering, $211(5), 345-355$.

Deb, K., Multi-objective Optimization using Evolutionary Algorithms, New York, NY, USA: John Wiley \& Sons, Inc. (2001).

Deb, K., Pratap, A., Agarwal, S., and Meyarivan, T. (2002), "A fast and elitist multiobjective genetic algorithm: NSGA-II," IEEE Transactions on Evolutionary Computation, 6(2), 182197.

Dreo, J., Siarry, P., Petrowski, A., and Taillard, E., Metaheuristics for Hard Optimization, New York: Springer-Verlg Berlin Heidelberg (2006).

Fleming, P.J., and Purshouse, R.C. (2002), "Evolutionary algorithms in control systems engineering: A survey," Control Engineering Practice, 10(11), 1223-1241.

Frank, P.M. (1990), "Fault diagnosis in dynamic systems using analytical and knowledge-based redundancy-a survey and some new results," Automatica, 26(3), 459-474.

Franklin, G.F., Powell, J.D., and Emami-Naeini, A., Feedback Control of Dynamic System, $4^{\text {th }}$ Edition, Upper Saddle River, NJ, USA: Prentice Hall, Inc. (2002).

Friedland, B., Control System Design - an introduction to state space methods, McGraw Hill (1986).

Friedland, B., Advanced Control System Design, Upper Saddle River, NJ, USA: Prentice-Hall Inc. (1996).

Goldberg, D.E., Genetic algorithm in Search, Optimisation and Machine Learning., Boston, MA, USA: Addison-Wesley Longman Publishing Co., Inc. (1989).

Goodall, R.M. (1994), "Dynamic characteristics in the design of MAGLEV suspensions," Proceedings of the Institution of Mechanical Engineers, Part F: Journal of Rail and Rapid Transit, 208(1), 33-41.

Goodall, R.M. (2000), "On the robustness of flux feedback control for electro-magnetic MAGLEV Controllers," in Proceedings of 16th International Conference on MAGLEV Systems and Linear Drives, pp. 197-202.

Goodall, R.M. (2004), "Dynamics and control requirements for EMS Maglev suspensions," in Proceedings on international conference on Maglev, pp. 926-934. 
Goodall, R.M. (2008), "Generalised Design Models for EMS Maglev," in Proceedings of MAGLEV 2008 - The $20^{\text {th }}$ International Conference on Magnetically Levitated Systems and Linear Drives.

Goodall, R.M. (Sep 1985), "The theory of electromagnetic levitation," Physics in Technology, 16(5), 207-213.

Isermann, R. (1997), "Supervision, fault-detection and fault-diagnosis methods-an introduction," Control Engineering Practice, 5(5), 639-652.

Isermann, R., and Ball, P. (1997), "Trends in the application of model-based fault detection and diagnosis of technical processes," Control Engineering Practice, 5(5), 709-719.

Kalman, R.E., and Bucy, R.S. (1961), "New Results in Linear Filtering and Prediction Theory," Random processes, 83(1), 95-108.

Konak, A., Coit, D.W., and Smith, A.E. (2006), "Multi-objective optimization using genetic algorithms: A tutorial," Reliability Engineering and System Safety, 91(9), 992-1007.

Lee, H.W., Kim, K.C., and Lee, J. (2006), "Review of Maglev train technologies," IEEE Transactions on Magnetics, 42(7), 1917-1925.

Maciejowski, J.M., Multivariable Feedback Design, Boston, MA: Addison-Wesley Longman Publishing Co. (1990).

Michail, K., "Optimised Configuration of Sensing Elements For Control And Fault Tolerance Applied To An Electro-Magnetic Suspension System," PhD Dessartation, Loughborough University, Department of Electronic and Electrical Engineering.http://hdl.handle.net/2134/5806 (2009).

Paddison, J.E., "Advanced Control Strategies for Maglev Suspension Systems," PhD Dissertation, Loughborough Univeristy, Department of Electronic and Electrical Engineering (1995).

Panagopoulos, H., Astrom, K.J., and Hagglund, T. (2002), "Design of PID controllers based on constrained optimisation," IEE Proceedings Control Theory and Applications, 149(1), $32-40$.

Patton, R.J. (1997), "Fault-Tolerant Control: The 1997 Situation," in IFAC Symposium on Fault Detection Supervision and Safety for Technical Processes, Vol. 3, pp. 1029-1052.

Skogestad, S., and Postlethwaite, I., Multivariable Feedback Control Analysis and Design, New York: John Wiley \& Sons Ltd, $2^{\text {nd }}$ Edition (2005).

Wal, M.V.D., and Jager, B.D. (2001), "A review of methods for input/output selection," Automatica, 37(4), 487-510.

Zou, X., Chen, Y., Liu, M., and Kang, L. (2008), "A New Evolutionary Algorithm for Solving Many-Objective Optimization Problems," IEEE Transactions on Systems, Man, and Cybernetics, Part B: Cybernetics, 38(5), 1402-1412. 